\title{
Insects galls of Pantanal areas in the State of Mato Grosso do Sul, Brazil: characterization and occurrence
}

\author{
SHARLENE ASCENDINO and VALÉRIA C. MAIA \\ Museu Nacional, Depto. Entomologia, Quinta da Boa Vista, s/n, São Cristóvão, 20940-040 Rio de Janeiro, RJ, Brazil
}

Manuscript received on July 24, 2017; accepted for publication on December 8, 2017

\begin{abstract}
Two Pantanal areas were investigated in Corumbá, Mato Grosso do Sul. The field works were carried out in five trails, in August and December/2011 and April/2012. Seventy-six gall morphotypes were found in 52 host plants, 22 of them were identified at species level and 30 at genus. Fabaceae, Myrtaceae and Sapindaceae were the plant families with the greatest richness of gall. Leaves were the plant organ with the greatest gall richness. Six gall shapes were found - globoid, lenticular, fusiform, cylindrical, conical, and marginal roll. The three first were the most common. Five colors were observed - green, brown, red, yellow, and black. Green and brown predominated. Most were glabrous and one-chambered. These features have been indicated in several Brazilian biomes. Diptera (Cecidomyiidae) were the most frequent gallers. The associated fauna comprised parasitoids (Eulophidae, Braconidae, Eupelmidae, Eurytomidae, Platygastridae, and Pteromalidae), inquilines (Cecidomyiidae: Trotteria Kieffer, 1902, Hymenoptera: Tanaostigmoides Ashmead, 1896, Hemiptera, Lepidoptera and Thysanoptera) and predators (Pseudoscorpionida: Olpiolum medium Beier, 1931, Cecidomyiidae: Lestodiplosini and Hymenoptera: Formicidae). Parasitoids were the most frequent. Multiparatism was observed. Our data represent the first contribution to the knowledge of the associated fauna in the Pantanal.
\end{abstract}

Key words: Associated fauna, forest capões, gall richness, host plants, riparian forest.

\section{INTRODUCTION}

Galls are atypical plant developments that provide shelter, food and protection to the inducing organism or its offspring (Shorthouse et al. 2005). They are induced by mites and insects, each species being responsible for the development of a morphologically and structurally unique gall in the host plant (Dreger-Jauffret and Shorthouse 1992). The galls can occur in any plant organ: roots,

Correspondence to: Sharlene Ascendino

E-mail: ascendinosh@gmail.com fruits, stems, flowers, leaves and others. They are classified according to their structural complexity as simple (without reorganization of the vegetal tissue) and complex (with reorganization of the vegetal tissue), to their morphology as clavate, conical, cylindrical, fusiform, globoid, lenticular, rosette, marginal roll, etc., to the number of internal chambers as one-chambered (with a single chamber) or multichambered (with two or more chambers) and in relation to the presence or absence of trichomes as glabrous or pubescent. Insect galls are induced by six orders: Coleoptera, 
Diptera, Hemiptera, Hymenoptera, Lepidoptera, and Thysanoptera (Mani 1964). A rich and frequent fauna of arthropods can be found in association with the galls, acting as parasitoids, predators, inquilines and/or successors.

Gall inventories in Brazil refer mainly to the Atlantic Forest and Cerrado in the Southeast Region (Maia 2013, Coelho et al. 2009). For the other Brazilian biomes and regions there is a lack of inventories and taxonomic studies. Julião et al. (2002) published the first insect gall inventory of the Pantanal (focus of the present study), where 133 gall morphotypes in 75 plant species were recorded. More recently, Urso-Guimarães et al. (2017) published a wide inventory of insect galls of the Mato Grosso do Sul, where 32 gall morphotypes in 23 plant species were recorded in Pantanal areas. These two studies surveyed the same Pantanal sites, the "Base de Estudos do Pantanal" (BEP) and the São Bento farm, both situated in the municipality of Corumbá, State of Mato Grosso do Sul (MS), central-western Brazil.

The Pantanal biome is the largest floodplain in the world, flooded most of the time and with periodic flooding (Oliveira 2007). With an area of about $150,000 \mathrm{~km}^{2}$, which occupies $1.8 \%$ of the Brazilian territory (IBGE 2004), it is considered the smallest Brazilian biome, influenced by three others: Amazonia, Cerrado, and Atlantic forest (MMA 2015). In Brazil, the Pantanal spreads over two States: Mato Grosso (MT) and Mato Grosso do Sul (MS). Although MS is smaller in territorial extent than MT, it comprises most of the Pantanal, $65.0 \%$ (IBGE 2004).

The present study aimed at increasing the knowledge of the diversity of insect galls in areas of the Pantanal, contributing to the taxonomic knowledge of galling insects and host plants in this biome and in Brazil, and adding new data on the associated fauna.

\section{MATERIALS AND METHODS}

Insect galls were collected in the municipality of Corumbá in the State of Mato Grosso do Sul in two localities (Fig. 1): Base de Estudos do Pantanal (BEP) and São Bento Farm (SBF). The BEP (S $19^{\circ} 34^{\prime} 57^{\prime}$ "W $\left.57^{\circ} 01^{\prime} 07^{\prime \prime}\right)$ is located on the banks of the Miranda River and consists of riparian forest (RF) (Fig. 2). The collections were carried out in three trails, in August and December of 2011 and April of 2012, totaling 72 hours. The São Bento Farm (SBF) consists of forest capões (FC), which are isolated formations of small agglomerates of the natural forest with closed arborous vegetation (Rambo 1956, Silva et al. 1998) (Fig. 3). Two collections were carried out in this locality: capão 1 (S 19 27'94" W $57^{\circ} 1^{\prime}$ '49") in August of 2011 and

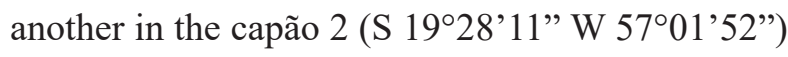
in April of 2012.

The vegetation was examined in search of galling insects and all plant organs were investigated, except subterranean roots. Fertile branches were collected from each host plant for the preparation of exsiccates. They were examined by botanists of Universidade Federal Rural da

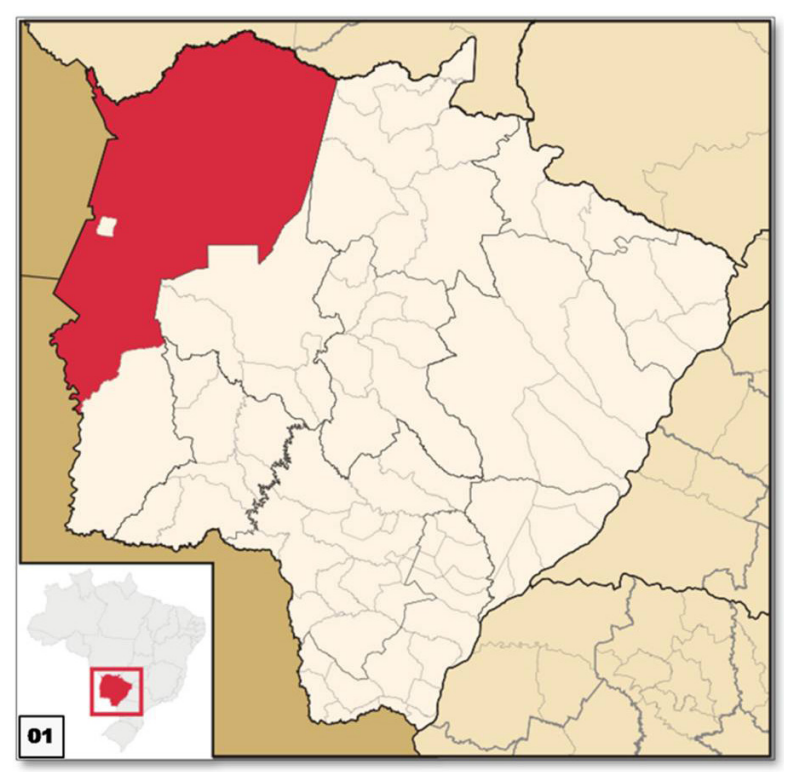

Figure 1 - Municipality of Corumbá in the State of Mato Grosso do Sul. 

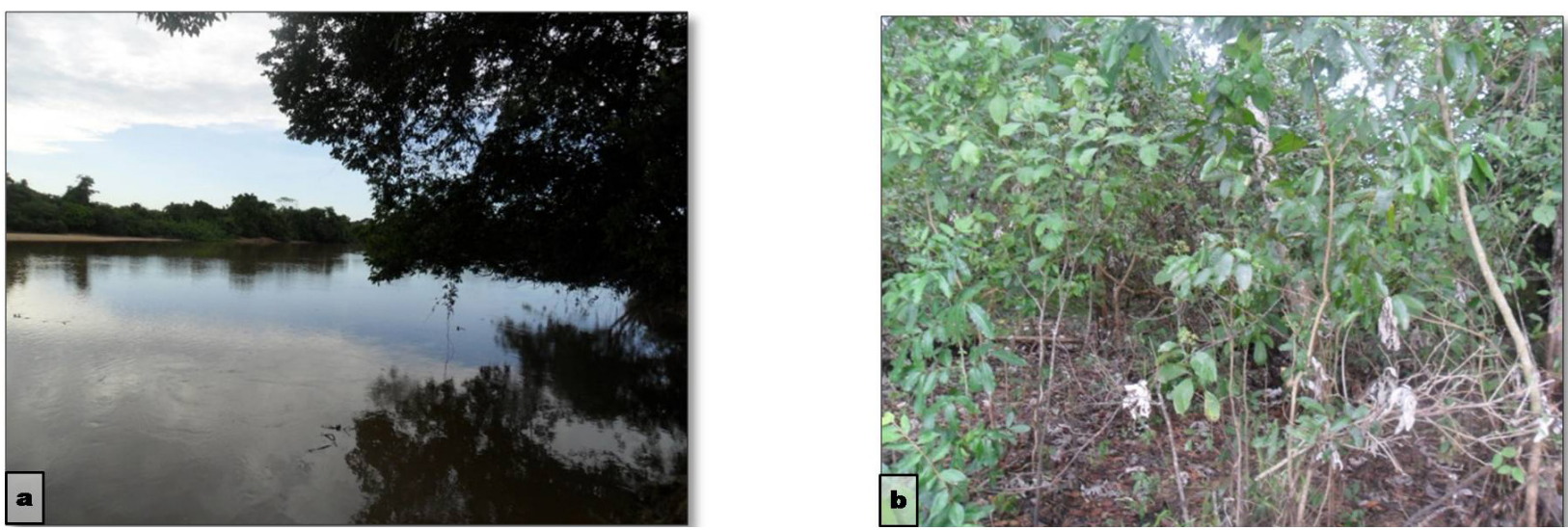

Figure 2 - Study sites in BEP (Base de Estudos do Pantanal): (a) banks of the Miranda River; (b) riparian forest of the banks of the River Miranda.
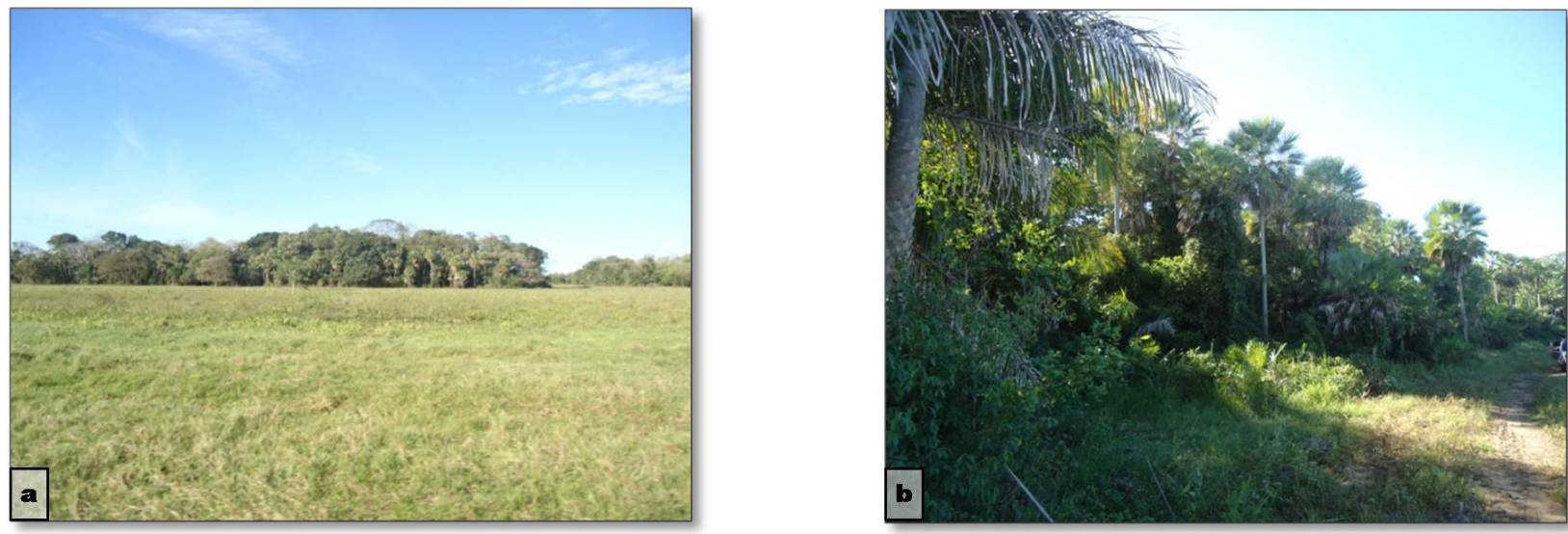

Figure 3 - Study sites in the SBF (São Bento Farm) (Corumbá, Mato Grosso do Sul, Brazil). (a) Capão 1; (b) Capão 2.

Amazônia (Pará) for species identification and the exsiccates were deposited in this Institution. Each gall morphotype was photographed in the field. Vegetable branches with galls were obtained and separated by host species and gall morphotype (to avoid mixing of material), labeled, numbered (the same number of the corresponding exsiccates) and were conditioned and transported in plastic bags to laboratory of Diptera of the Department of Entomology (Museu Nacional do Rio de Janeiro/ MNRJ-UFRJ). Part of the material was conditioned in closed transparent plastic pots, labeled and lined with humid paper. These pots were examined daily for emergence of adult insects (inducers, parasitoids, inquilines, predators and successors). The other part of the sample was dissected with the use of stereoscopic microscope to obtain the immature stages and determine the number of internal chambers.

All insects were determined preliminarily in order and/or family and preserved in $70 \%$ alcohol. Cecidomyiidae (Diptera) were later mounted on microscope slides following the methodology outlined in Gagné 1994, the genera were identified using the keys of Gagné 1994 and the species based on their original descriptions. They were deposited in the Entomology collection of the Museu Nacional of Rio de Janeiro (MNRJ)/UFRJ. The other arthropods were identified in the lowest possible taxonomic level by specialists: Pseudoscorpionida by Dr. Mark Harvey (Western Australian Museum), Coleoptera by Dr. Paulo Magno (Museu Nacional, 
UFRJ) and Hymenoptera by Dra. Maria Antonieta Pereira de Azevedo (Museu Nacional, UFRJ) and the specimens were deposited in the scientific collections of the institutions of these specialists. The other insects (Hemiptera and Lepidoptera) were identified only at order level.

The botanical names were updated using the Flora do Brasil website (Flora do Brasil 2020). The geographic distribution of the host plant species in Brazil, as well as data on endemism were retrieved from this same website.

\section{RESULTS}

Seventy-six gall morphotypes were found in 52 host plants, 22 of them were identified at species level and 30 at genus (Table I). All host plant species are native, among them, three are endemic in Brazil: Andira inermis (W.Wright) Kunth ex DC, Blanchetiodendron blanchetii (Benth.) Barneby and J.W. Grimes, both belong to Fabaceae and Smilax brasiliensis Spreng (Smilacaceae). These endemic plants hosted four, one and one gall morphotypes, respectively, totaling six.

Seven plant species are recorded for the first time in the State of Mato Grosso do Sul: Blanchetiodendron blanchetii,Inga ingoides (Rich.) Willd., Zygia latifolia var. communis Barneby and J.W.Grimes (Fabaceae), Vitex schomburgkiana Schauer (Lamiaceae), Calyptranthes nigricans DC. (Myrtaceae), Salix humboldtiana Willd. (Salicaceae) and Smilax irrorata Mart. ex Griseb. (Smilacaceae).

Pfaffia glomerata (Spreng.) Pedersen (Amaranthaceae), Annona cf. tenuipes R. E. Fr. (Annonaceae), Dolichandra unguis-cati (L.) L. G. Lohmann (Bignoniaceae), Inga ingoides (Rich.) Willd (Fabaceae), Trichilia quadrijuga Kunth (Meliaceae), Calyptranthes nigricans DC. (Myrtaceae), Eugenia flavescens DC (Myrtaceae), Coccoloba diversifolia Jacq. (Polygonaceae), Casearia aculeata Jacq. (Salicaceae), Paullinia ingifolia Rich. ex Juss (Sapindaceae), Smilax brasiliensis Spreng, Smilax irrorata Mart. ex Griseb (Smilacaceae) and Lippia alba (Mill.) N. E. Br. ex Britton and P. Wilson (Verbenaceae) are recorded for the first time as host plants of galling insects in the Pantanal. Most of them presented a single gall morphotype, except Inga ingoide with two and Paullinia ingifolia and Eugenia flavescens, both with three, totaling 18 gall morphotypes.

The mean number of gall morphotypes per plant morphospecies was 1.46. Fabaceae, Myrtaceae and Sapindaceae were the plant families with the greatest richness of gall, with 15, 13 and nine gall morphotypes, distributed in ten, eight and three plant species, respectively.

Leaves were the plant organ with the greatest gall richness in both localities, with 58 morphotypes, totaling $76.3 \%$ (75.4\% in the BEP and $81.2 \%$ in the SBF), followed by stems with 15 morphotypes, $19.7 \%$ (22.9\% in BEP and $12.5 \%$ in SBF), and buds with three morphotypes, 3.9\% $(1.75 \%$ in BEP and $12.5 \%$ in $\mathrm{SBF})$.

Concerning the gall external morphology, six shapes were found - globoid, lenticular, fusiform, cylindrical, conical, and marginal roll. The three first were the most common, with $38.1 \%$ (36.0\% in the BEP and $43.7 \%$ in the SBF), $25.0 \%$ (26.2\% in the BEP and $25.0 \%$ in the SBF), and $17.1 \%(19.6 \%$ in the BEP and $6.2 \%$, respectively. All other shapes presented lower percentages (less than 10.0\%): cylindrical (9.8\% in the BEP, not found in the SBF), conical $(8.1 \%$ in the BEP and $6.25 \%$ in the SBF), and marginal roll (not found in the BEP and 6.2\% in the SBF). Five colors were observed -green, brown, reddish, yellow, and black. The green color predominated, with $43.4 \%$ (44.2\% in the BEP and $37.5 \%$ in the SBF), followed by brown, with $35.5 \%$ (36.0\% inthe BEP and $37.5 \%$ in the BEP, with one morphotype found in both localities). The other together represented less than $30.0 \%$ of the total: yellow (14.7\% in the BEP and $25.0 \%$ in the SBF), reddish and black, both colors found only in the 
TABLE I

Richness of gall morphotypes by host plant family and species in two sites of Corumbá, biome Pantanal, Mato Grosso do Sul (Midwestern Brazil). BEP - Base de Estudos do Pantanal, RF - Riparian Forest, SBF - São Bento Farm, FC - Forest Capões.

\begin{tabular}{|c|c|c|c|}
\hline $\begin{array}{l}\text { Host plant family } \\
\qquad(\mathrm{n}=\mathbf{2 3})\end{array}$ & $\begin{array}{l}\text { Host plant species } \\
\qquad(\mathrm{n}=\mathbf{5 8})\end{array}$ & $\begin{array}{c}\text { Nr. of gall } \\
\text { morphotypes } \\
(n=83)\end{array}$ & Site \\
\hline \multirow[t]{2}{*}{ Amaranthaceae A. Juss } & Pfaffia glomerata (Spreng.) Pedersen & 01 & $\mathrm{BEP}(\mathrm{RF})$ \\
\hline & Total & 01 & \\
\hline \multirow[t]{2}{*}{ Annonaceae Juss. } & Annona cf. tenuipes R.E.Fr. & 01 & $\mathrm{BEP}(\mathrm{RF})$ \\
\hline & Total & 01 & \\
\hline \multirow{5}{*}{ Bignoniaceae Juss. } & Dolichandra unguis-cati (L.) L.G.Lohmann & 01 & $\mathrm{BEP}(\mathrm{RF})$ \\
\hline & Dolichandra sp. & 01 & $\mathrm{BEP}(\mathrm{RF})$ \\
\hline & Fridericia sp. & 01 & $\mathrm{SBF}(\mathrm{FC})$ \\
\hline & Tabebuia sp. & 01 & $\mathrm{BEP}(\mathrm{RF})$ \\
\hline & Total & 04 & \\
\hline \multirow[t]{2}{*}{ Cannabaceae Martinov } & Celtis cf. iguanaea (Jacq.) Sarg. & 01 & $\mathrm{BEP}(\mathrm{RF})$ \\
\hline & Total & 01 & \\
\hline \multirow[t]{2}{*}{ Celastraceae R.Br. } & Prionostemma sp. & 01 & $\mathrm{BEP}(\mathrm{RF})$ \\
\hline & Total & 01 & \\
\hline \multirow[t]{3}{*}{ Chrysobalanaceae R.Br. } & Licania sp. 1 & 01 & $\mathrm{SBF}(\mathrm{FC})$ \\
\hline & Licania sp. 2 & 01 & $\mathrm{SBF}(\mathrm{FC})$ \\
\hline & Total & 02 & \\
\hline \multirow[t]{3}{*}{ Combretaceae R.Br. } & Combretum sp. 1 & 01 & $\mathrm{BEP}(\mathrm{RF})$ \\
\hline & Combretum sp. 2 & 03 & $\mathrm{BEP}(\mathrm{RF})$ \\
\hline & Total & 04 & \\
\hline \multirow[t]{2}{*}{ Euphorbiaceae Marc.-Berti } & Sebastiania sp. & 02 & $\mathrm{SBF}(\mathrm{FC})$ \\
\hline & Total & 02 & \\
\hline \multirow[t]{12}{*}{ Fabaceae Lindl. } & Aeschynomene sp. & 02 & $\mathrm{BEP}(\mathrm{RP})$ \\
\hline & Andira cf. inermis (W.Wright) Kunth ex DC & 01 & $\mathrm{SBF}(\mathrm{FC})$ \\
\hline & Total & 01 & \\
\hline & Andira inermis (W.Wright) Kunth ex DC & 04 & $\mathrm{BEP}(\mathrm{RP})$ \\
\hline & Blanchetiodendron blanchetii (Benth.) Barneby and J.W. Grimes & 01 & $\mathrm{BEP}(\mathrm{RP})$ \\
\hline & Copaifera sp. & 02 & $\mathrm{SBF}(\mathrm{FC})$ \\
\hline & Derris sp. & 01 & $\mathrm{BEP}(\mathrm{RP})$ \\
\hline & Inga ingoides (Rich.) Willd & 02 & $\mathrm{BEP}(\mathrm{RP})$ \\
\hline & Inga sp. & 01 & $\mathrm{SBF}(\mathrm{FC})$ \\
\hline & Mimosa tweedieana Barneby ex Glazier and Mackinder & 01 & $\mathrm{BEP}(\mathrm{RP})$ \\
\hline & Zygia latifolia var. communis Barneby and J.W.Grimes & 01 & $\mathrm{BEP}(\mathrm{RP})$ \\
\hline & Total & 15 & \\
\hline \multirow[t]{3}{*}{ Lamiaceae Martinov } & Hyptis sp. & 01 & $\mathrm{SBF}(\mathrm{FC})$ \\
\hline & Vitex schomburgkiana Schauer & 01 & $\mathrm{BEP}(\mathrm{RP})$ \\
\hline & Total & 02 & \\
\hline
\end{tabular}


TABLE I (continuation)

\begin{tabular}{|c|c|c|c|c|}
\hline $\begin{array}{l}\text { Host plant family } \\
\qquad(\mathrm{n}=\mathbf{2 3})\end{array}$ & $\begin{array}{l}\text { Host plant species } \\
\qquad(n=58)\end{array}$ & & $\begin{array}{c}\text { Nr. of gall } \\
\text { morphotypes } \\
(n=83)\end{array}$ & Site \\
\hline \multirow[t]{2}{*}{ Malpighiaceae Juss. } & Byrsonima sp. (L.) Kunth & & 02 & $\mathrm{BEP}(\mathrm{RP})$ \\
\hline & & Total & 02 & \\
\hline \multirow{2}{*}{ Meliaceae A. Juss. } & Trichilia quadrijuga Kunth & & 01 & $\mathrm{BEP}(\mathrm{RP})$ \\
\hline & & Total & 01 & \\
\hline \multirow[t]{3}{*}{ Moraceae Gaudich. } & Ficus sp. 1 & & 01 & $\mathrm{BEP}(\mathrm{RP})$ \\
\hline & Ficus sp. 2 & & 01 & $\mathrm{SBF}(\mathrm{FC})$ \\
\hline & & Total & 02 & \\
\hline \multirow[t]{9}{*}{ Myrtaceae Juss. } & Calyptranthes nigricans DC. & & 01 & SBF (FC) \\
\hline & Calyptranthes sp. & & 01 & $\begin{array}{l}\text { SBF }(F C) \\
\text { and BEP } \\
\text { (RP) }\end{array}$ \\
\hline & Eugenia flavescens DC & & 03 & $\mathrm{BEP}(\mathrm{RP})$ \\
\hline & Eugenia sp. 1 & & 03 & $\mathrm{BEP}(\mathrm{RP})$ \\
\hline & Eugenia sp. 2 & & 01 & $\mathrm{BEP}(\mathrm{RP})$ \\
\hline & Marlierea sp. & & 01 & $\mathrm{BEP}(\mathrm{RP})$ \\
\hline & Myrcia sp. & & 01 & $\mathrm{BEP}(\mathrm{RP})$ \\
\hline & Psidium sp. & & 02 & $\mathrm{BEP}(\mathrm{RP})$ \\
\hline & & Total & 13 & \\
\hline \multirow[t]{2}{*}{ Phyllanthaceae Martinov } & Phyllanthus sp. & & 01 & $\mathrm{BEP}(\mathrm{RP})$ \\
\hline & & Total & 01 & \\
\hline \multirow[t]{5}{*}{ Polygonaceae A. Juss. } & Coccoloba diversifolia Jacq. & & 01 & $\mathrm{BEP}(\mathrm{RP})$ \\
\hline & Coccoloba sp. 1 & & 02 & $\mathrm{BEP}(\mathrm{RP})$ \\
\hline & Coccoloba sp. 2 & & 02 & $\mathrm{SBF}(\mathrm{FC})$ \\
\hline & Coccoloba sp. 3 & & 03 & $\mathrm{BEP}(\mathrm{RP})$ \\
\hline & & Total & 09 & \\
\hline \multirow[t]{2}{*}{ Rutaceae A. Juss. } & Zanthoxylum sp. & & 01 & $\mathrm{BEP}(\mathrm{RP})$ \\
\hline & & Total & 01 & \\
\hline \multirow[t]{3}{*}{ Salicaceae Mirb. } & Casearia aculeata Jacq. & & 01 & $\mathrm{BEP}(\mathrm{RP})$ \\
\hline & Salix humboldtiana Willd. & & 01 & $\mathrm{BEP}(\mathrm{RP})$ \\
\hline & & Total & 02 & \\
\hline \multirow[t]{4}{*}{ Sapindaceae Juss. } & Paullinia ingifolia Rich. ex Juss & & 04 & $\mathrm{BEP}(\mathrm{RP})$ \\
\hline & Paullinia pinnata $\mathrm{L}$. & & 05 & $\mathrm{BEP}(\mathrm{RP})$ \\
\hline & Vouarana sp. & & 01 & $\mathrm{BEP}(\mathrm{RP})$ \\
\hline & & Total & 10 & \\
\hline \multirow[t]{4}{*}{ Smilacaceae Vent. } & Smilax brasiliensis Spreng. & & 01 & $\mathrm{BEP}(\mathrm{RP})$ \\
\hline & Smilax irrorata Mart. ex Griseb & & 01 & $\mathrm{BEP}(\mathrm{RP})$ \\
\hline & Smilax sp. & & 01 & $\mathrm{BEP}(\mathrm{RP})$ \\
\hline & & Total & 03 & \\
\hline \multirow[t]{2}{*}{ Verbenaceae J.St.-Hil } & Lippia alba (Mill.) N.E.Br. ex Britton and P.Wilson & & 01 & $\mathrm{BEP}(\mathrm{RP})$ \\
\hline & & Total & 01 & \\
\hline
\end{tabular}


BEP, with $3.28 \%$ and $1.64 \%$, respectively. Most galls $(82.8 \%)$ were glabrous $(85.2 \%$ in the BEP and $75.0 \%$ in the SBF). Concerning the internal morphology, $80.2 \%$ were one-chambered $(81.9 \%$ in the BEP and $75.0 \%$ in the SBF).

The inducers of 40 gall morphotypes (52.6\%) were determined $(52.5 \%$ in the BEP and $56.3 \%$ in the SBF). They belong to three insect orders: Diptera (Cecidomyiidae) responsible for 35 gall morphotypes, Hemiptera (four) and Coleoptera (one). Among the determined gallers, most were Cecidomyiidae (44.2\% in the BEP and $56.2 \%$ in the SBF). Hemipteran and coleopteran galls were found only in the BEP. Cecidomyiidae were represented by two supertribes: Lasiopteridi and Cecidomyiidi. Among the former, the genus Neolasioptera Felt, 1908 was identified (with five morphospecies in the BEP and a single one in the $\mathrm{SBF}$ ). Among the latter, three tribes were recorded: Asphondyliini (Schizomyiina), Clinodiplosini, Lopesiini, all obtained in the BEP and represented by a single morphospecies. The other gall midges were identified at family level.

The associated fauna comprised parasitoids, inquilines, and predators (Table SII - Supplementary Material). The parasitoids were the most frequent. They were found in 22 gall morphotypes (28.9\%) and included 21 morphospecies distributed in five families of Hymenoptera: Eulophidae (eight morphospecies), Braconidae(three morphospecies), Eupelmidae (three morphospecies), Eurytomidae (four morphospecies + a single pupa which could not be morphospecified), Platygastridae (one morphospecies), and Pteromalidae (two morphospecies). Among them, Eulophidae were the most frequent. Besides these records, other seven parasitized gall morphotypes were found, but as the Hymenoptera were obtained only as larva, they could not be separated in morphospecies.

Multiparatism was found in six gall morphotypes, namely: globoid leaf gall on Myrcia sp., lenticular leaf gall on Coccoloba sp.1, globoid leaf gall on Coccoloba sp.2, globoid leaf gall on Salix humboltiana, globoid leaf gall on Paullinia ingifolia and lenticular leaf gall on Smilxa irrorata. From each morphotype, two species of wasps were obtained.

The parasitoids were obtained mainly from leaf $(86.9 \%)$, but also from stem galls $(13.0 \%)$. If we consider the total number of leaf galls, $34.5 \%$ of them were parasitized. Similarly, if we consider the total number of stem galls, $20.0 \%$ were parasitized. Most parasitoids occurred in glabrous galls (82.6\%), but some of them were obtained from pubescent galls $(17.4 \%)$. The parasitized glabrous galls represented $30.1 \%$ of the total of the glabrous galls, and the parasitized pubescent galls represented $30.8 \%$ of the total of the pubescent galls. The parasitoids were found in four gall shapes, globoid, lenticular, fusiform, conical, being most frequent in globoid galls (43.5\%). Ten plant families hosted parasitoids, among them, Fabaceae, Myrtaceae, Sapindaceae and Polygonaceae comprised together $65.2 \%$ of the records.

The inquilines, found in five gall morphotypes (6.5\%), were represented by four insect orders: Trotteria Kieffer, 1902 (Cecidomyiidae, Diptera) (one morphospecies), Tanaostigmoides Ashmead, 1896 (Tanaostigmatidae, Hymenoptera) (one morphospecies), Hemiptera (two morphospecies), Lepidoptera (one morphospecies) and Thysanoptera (one morphospecies). Two gall morphotypes comprised two different inquilines: Lepidoptera + Hemiptera (on Tabebuia sp., Bignoniaceae) and Lepidoptera + Trotteria sp. (Cecidomyiidae) (on Vouarana sp., Sapindaceae), the others a single one.

Two different guilds of dwellers were obtained from a single gall morphotype on Paullinia ingifolia (Sapindaceae): Tanaostigmoides sp.1 (inquiline) and Eurytomidae sp.3 and Braconidae sp.3 (parasitoids).

The predators were represented by Olpiolum medium Beier, 1931 (Olpiidae: Pseudoscorpionida), 
Lestodiplosini: Cecidomyiidae (one morphospecies), andFormicidae:Hymenoptera(onemorphospecies).

The associated fauna of the BEP was richer than that of the SBF. In the BEP, it was composed by inquilines - Trotteria sp., Tanaostigmoides sp., Hemiptera sp., Thysanoptera sp. and Lepidoptera sp.; parasitoids (Hymenoptera) - Braconidae (sp. 1 and sp. 3), Eulophidae (sp.1-sp.8), Eupelmidae (sp. 2 and sp.3), Eurytomidae (sp.1, sp.2, sp.3, sp.4), pupa and Sycophyla sp., Pteromalidae (sp. 1 and sp. 2), and Platygastridae sp.; and predators - Olpiolum medium, Formicidae sp. and Lestodiplosini. Whereas in the SBF, the associated fauna comprised only parasitoids - Braconidae sp. 2 and Eupelmidae sp. 1, both not found in the BEP.

Comparing the two studied physiognomies, we can realize that the BEP comprised greater gall richness than the SBF. In the former, we found 61 gall morphotypes in 41 host plants, 20 of them were identified at species level and 21 at genus level; and the medium number of gall morphotypes per plant morphospecies was 1.4. In the latter, we found 16 morphotypes in 12 host plants, only two identified at species level and 10 at genus; and a similar medium number of gall morphotypes per plant morphospecies (1.3). A single gall morphotype occurred in both physiognomies on Calyptranthes sp. (Myrtaceae). In the BEP, Myrtaceae and Fabaceae were the superhost families, the former with eight galled species each. Myrtaceae hosted 12 gall morphotypes, whereas Fabaceae 11. In the SBF no plant family comprised more than four galls. Because of it, no superhost family was pointed out.

Data on galls morphology, inducers, associated fauna, sites and dates of collection are presented below. The data were organized in alphabetical order by family plant, genus and species. The number of gall morphotypes for each botanical taxon is given in brackets. The previous gall records in each plant species or genus in Brazil were indicated, as well as the new records.
Amaranthaceae A. Juss. (new record of host plant family) $(\mathrm{n}=1)$

Pfaffia glomerata (Spreng.) Pedersen ( $\mathrm{n}=1)$

Gall (Fig. 4): on stem, globoid, brown, glabrous, one-chambered. Galler: Undetermined. Site: BEP (RF), August/2011.

Annonaceae Juss. $(\mathrm{n}=1)$

Annona cf. tenuipes R.E.Fr. $(\mathrm{n}=1)$

Gall (Fig. 5): on leaf (adaxial surface), cylindrical, green, pubescent. Galler: Hemiptera. Site: BEP (RF), August/2011. Previous records on this plant species: Almada and Fernandes 2011 - lenticular leaf gall, Oriximiná (Pará). New gall morphotype.

Bignoniaceae Juss. $(\mathrm{n}=4)$

Fridericia $\mathrm{sp} .(\mathrm{n}=1)$

Gall (Fig. 6): on leafvein (abaxial surface), fusiform, green, glabrous, one-chambered. Galler: Undetermined. Other insects: Eupelmidae sp. 1 (parasitoid). Site: SBF (FC), April/2012. Previous records on Fridericia Mart. (= Arrabidaea DC.): Fernandes et al. 2001, Vale do Rio Doce (MG); Julião et al. 2002, Pantanal Sul-Mato-Grossense (MS), Fernandes et al. 1997, Vale do Jequitinhonha (MG), Maia 2001, Carapebus and Maricá (RJ), Oliveira and Maia 2005, Grumari (RJ).

Dolichandra unguis-cati (L.) L.G.Lohmann (new record de host plant) $(\mathrm{n}=1)$

Gall (Fig. 7): on stem, fusiform, brown, glabrous, one-chambered. Galler: Undetermined. Other insects: Hymenoptera (parasitoid). Site: BEP (RP), August/2011.

Dolichandra sp. (= Macfadyena sp.) $(\mathrm{n}=1)$

Gall (Fig. 8): on leaf (abaxial surface), cylindrical, yellow, glabrous, one-chambered. Galler: Undetermined. Site: BEP (RP), April/2012. Previous records on Dolichandra Cham.: Maia 2013 as Macfadyena sp., São Tomé das Letras (MG). New gall morphotype.

\section{Tabebuia sp. $(\mathrm{n}=1)$}

Gall (Fig. 9): on leaf (abaxial surface), globoid, yellow, glabrous, solid. Galler: Hemiptera. Other 

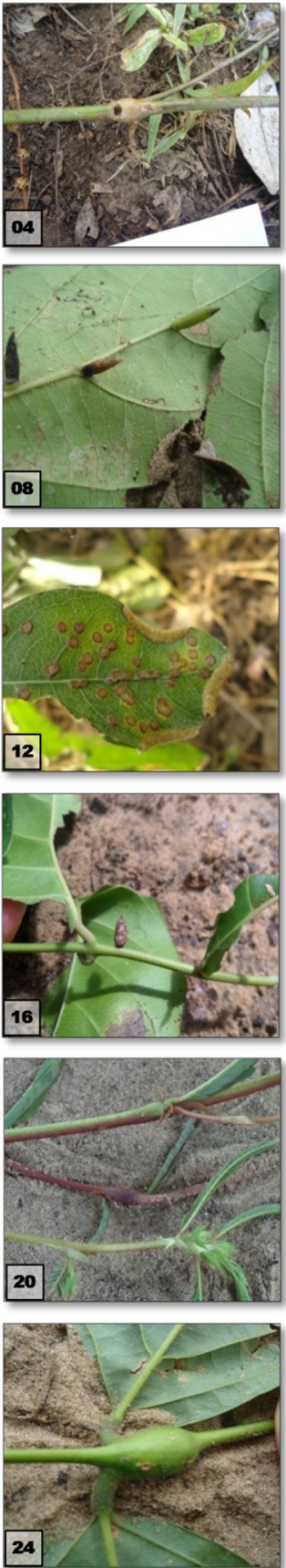
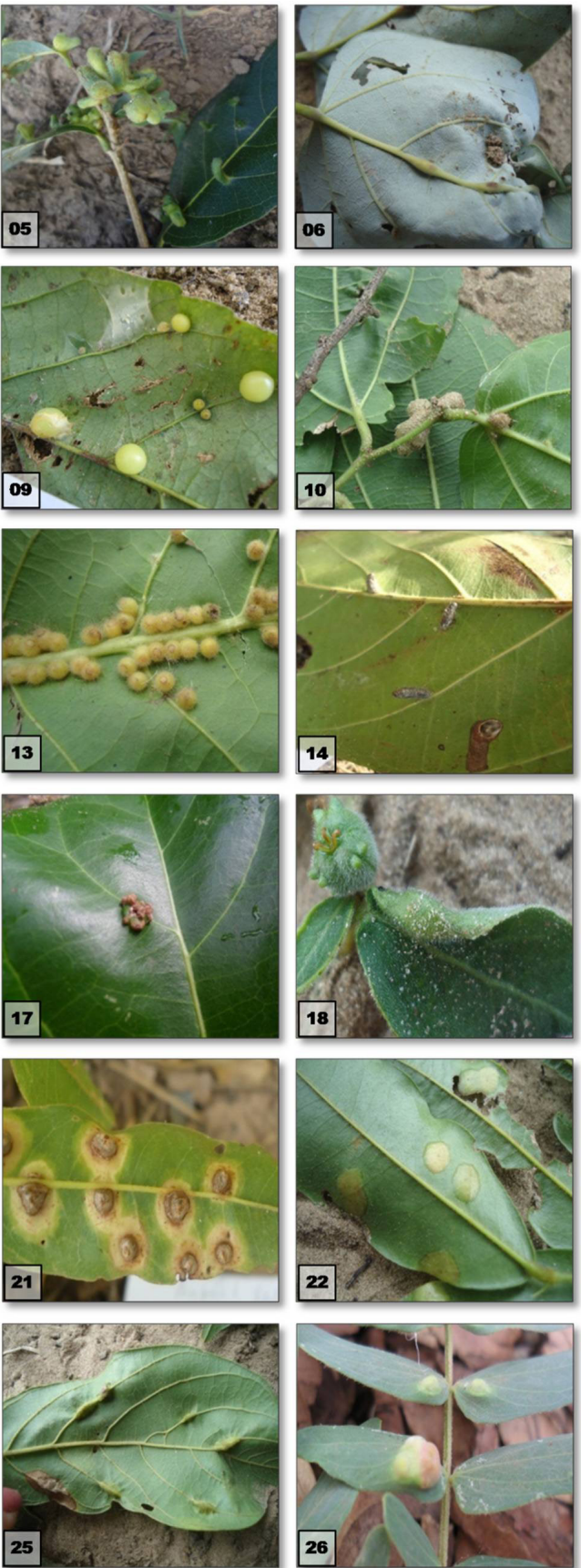
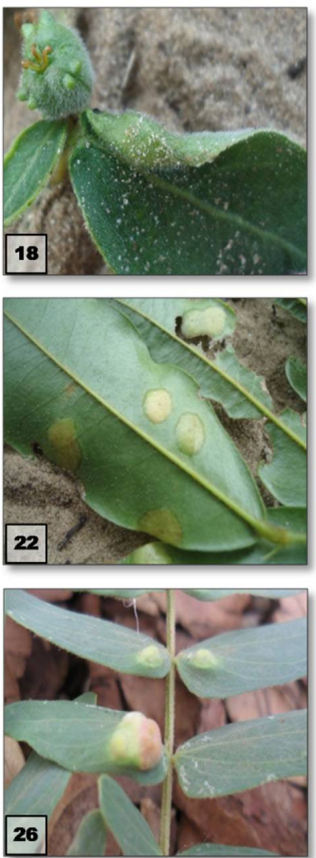
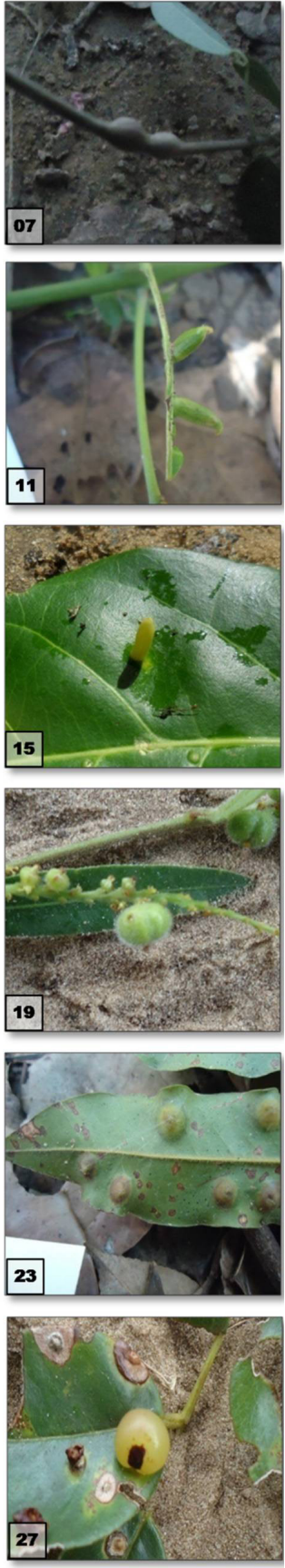

Figures 4-27 - (4) On Pfaffia glomerata: globoid stem gall; (5) On Annona cf. tenuipes: cylindrical leaf gall; (6) On Fridericia sp.: fusiform leafvein gall; (7) On Dolichandra unguis-cati: fusiform stem gall; (8) On Dolichandra sp. : cylindrical leaf gall; (9) On Tabebuia sp.: globoid leaf gall; (10) On Celtis cf. iguanaea: globoid leaf petiole gall; (11) On Prionostemma sp.: conical leaf gall; (12) On Licania sp. 1: marginal roll leaf gall; (13) On Licania sp. 2: globoid leaf gall; (14) On Combretum sp. 1: cylindrical leaf gall; (15-17): On Combretum sp. 2: (15) cylindrical leaf gall; (16) cylindrical stem gall; (17) lenticular leaf gall; (18-19) On Sebastiania sp.: (18) marginal roll leaf gall; (19) globoid bud; (20) On Aeschynomene sp.: fusiform stem gall; (21) On Andira cf. inermis: conical leaf gall; (22-25) On Andira inermis: (22) lenticular leaf gall; (23) lenticular leaf gall; (24) fusiform stem gall; (25) fusiform leafvein gall; (26) On Blanchetiodendron blanchetii: lenticular leaf gall; (27) On Copaifera sp.: globoid leaf gall. 
insects: Lepidoptera and Coccoidea (Hemiptera) (inquilines). Site: BEP(RP), August/2011. Previous records on Tabebuia Gomes ex DC.: CarvalhoFernandes et al. 2012, Região Xingó (PE), Araújo et al. 2011, Serra dos Pireneus (GO), Santos et al. 2012a, Caldas Novas (GO), Urso-Guimarães et al. 2003, Delfinópolis (MG), Fernandes et al. 1988, Belo Horizonte (MG), Coelho et al. 2013, Serra do Cabral, (MG), Carneiro et al. 2009, Cadeia do Espinhaço (MG), Maia et al. 2008, Bertioga (SP), Julião et al. 2002, Corumbá (MS), Fernandes et al. 1997, Vale do Jequitinhonha (MG).

Cannabaceae Martinov ( $\mathrm{n}=1)$

Celtis cf. iguanaea (Jacq.) Sarg. ( $\mathrm{n}=1)$

Gall (Fig. 10): on leaf petiole, globoid, brown, glabrous, one-chambered. Galler: Cecidomyiidae (Diptera). Site: BEP (RP), April/2012. New gall morphotype.

Previous records on this plant species: Santos et al. 2011, as Celtis membranacea Miq., Pernambuco.

Celastraceae R.Br. $(\mathrm{n}=1)$

Prionostemma sp. (new record of host plant genus) $(\mathrm{n}=1)$

Gall (Fig. 11): on leaf (adaxial surface), conical, green, glabrous, one-chambered. Galler: Cecidomyiidi (Diptera). Site: BEP (RP), August/2011.

Chrysobalanaceae R.Br. $(\mathrm{n}=2)$

Licania sp. $1(\mathrm{n}=1)$

Gall (Fig. 12): on leaf, marginal roll, brown, pubescent, one-chambered. Galler: Cecidomyiidae (Diptera). Site: SBF (FC), August/2011.

Licania $\mathrm{sp} .2(\mathrm{n}=1)$

Gall (Fig. 13): on leaf (abaxial surface), globoid, yellow, pubescent, one-chambered. Galler: Cecidomyiidae (Diptera). Site: SBF (FC), August/2011. Previous records on Licania Aubl.: Coelho et al. 2013, on Licania sp., Serra do Cabral (MG), Araújo et al. 2007, on Licania tomentosa (Benth.) Fritsch., Campus Samambaia (GO).

Combretaceae R.Br. $(n=4)$
Combretum sp. $1(\mathrm{n}=1)$

Gall (Fig. 14): on leaf (abaxial surface), cylindrical, brown, glabrous, one-chambered. Galler: Undetermined. Site: BEP (RP), August/2011. New gall morphotype.

Combretum sp. $2(\mathrm{n}=3)$

Gall (Fig. 15): on leaf (adaxial surface), cylindrical, green, glabrous, one-chambered. Galler: Cecidomyiidae (Diptera). Site: BEP (RP), December/2011. New gall morphotype.

Gall (Fig. 16): on stem, cylindrical, brown, glabrous, one-chambered. Galler: Undetermined. Site: BEP (RP), December/2011.

Gall (Fig. 17): on leaf (adaxial surface), lenticular, brown, glabrous, one-chambered. Galler: Undetermined. Site: BEP (RP), December/2011. Previous records on Combretum Loefl.: Tavares 1925, on Combretum leprosum Mart, Ceará, Almada and Fernandes 2011, on Combretum sp., Oriximiná (PA).

Euphorbiaceae Marc.-Berti ( $\mathrm{n}=2)$

Sebastiania sp. $(\mathrm{n}=2)$

Gall (Fig. 18): on leaf, marginal roll, green, glabrous, one-chambered. Galler: Cecidomyiidae (Diptera). Site: SBF (FC), April/2012. Previous records on Sebastiania Spreng: Maia 2001, on $S$. glandulosa (Mart.) Pax (heterotypic synonym of Microstachys corniculata (Vahl) Griseb.), Maricá (RJ).

Gall (Fig. 19): on bud, globoid, green, glabrous, multichambered. Galler: Cecidomyiidae (Diptera). Site: SBF (FC), April/2012. Previous records on Sebastiania spp.: Monteiro et al. 2004, on $S$. glandulosa (Mart.) Pax (heterotypic synonym of Microstachys corniculata (Vahl) Griseb.), Parque Nacional da Restinga de Jurubatiba (RJ).

Fabaceae Lindl. $(\mathrm{n}=15)$

Aeschynomene $\mathrm{sp} .(\mathrm{n}=1)$

Gall (Fig. 20): on stem, fusiform, reddish, glabrous, one-chambered. Galler: Neolasioptera sp. (Cecidomyiidae, Diptera). Site: BEP (RP), April/2012. 
Previous records: Maia et al. 2017, on Aeschynomene denticulata Rudd., Corumbá (MS).

Andira cf. inermis (W.Wright) Kunth ex DC $(\mathrm{n}=1)$

Gall (Fig. 21): on leaf (abaxial surface), conical, brown, glabrous. Galler: Undetermined. Site: SBF (FC), August/2011.

Andira inermis (W.Wright) Kunth ex DC ( $\mathrm{n}=4)$

Gall (Fig. 22): on leaf (abaxial and adaxial surfaces), lenticular, yellow, glabrous, one-chambered. Galler: Neolasioptera sp. (Cecidomyiidae, Diptera). Site: BEP (RP), April/2012. Previous records on this plant species: Julião et al. 2002, Corumbá (MS).

Gall (Fig. 23): on leaf (abaxial surface), lenticular, green, glabrous, one-chambered. Galler: Undetermined. Other insects: Braconidae sp. 1 (Hymenoptera) (parasitoid). Site: BEP (RP), August/2011. New gall morphotype.

Gall (Fig. 24): on stem, fusiform, green, glabrous, one-chambered. Galler: Undetermined. Other insects: Olpiolum medium Beier, 1931 (Olpiidae, Pseudoscorpiones) (predator). Site: BEP (RP), April/2012. New gall morphotype.

Gall (Fig. 25): on leafvein (abaxial surface), fusiform, green, glabrous, one-chambered. Galler: Undetermined. Other insects: Eulophidae sp. 1 (Hymenoptera) (parasitoid) and Hemiptera. Site: BEP (RP), April/2012. New gall morphotype.

Blanchetiodendron blanchetii (Benth.) Barneby and J.W. Grimes (new record of host plant species $)(n=1)$

Gall (Fig. 26): on leaf (adaxial surface), lenticular, green, glabrous, one-chambered. Galler: Cecidomyiidae (Diptera). Site: BEP (RP), December/2011.

Copaifera $\mathrm{sp} .(\mathrm{n}=2)$

Gall (Fig. 27): on leaf (adaxial surface), globoid, yellow, glabrous, one-chambered. Galler: Undetermined. Site: SBF (FC), April/2012.

Gall (Fig. 28): on leaf (adaxial surface), lenticular, yellow, glabrous, one-chambered.
Galler: Cecidomyiidae (Diptera). Site: SBF (FC), April/2012. Previous records on Copaifera Lindl.: Fernandes et al. 1988, on Copaifera sp., Belo Horizonte (MG), Maia and Fernandes 2004, Serra de São José (MG), Maia 2013, São Tomé das Letras (MG), Fernandes et al. 1988, Belo Horizonte (MG), Urso-Guimarães and Scarelli-Santos 2006, Reserva Pé de Gigante (SP), all records on $C$. langsdorffii Desf..

Derris sp. $(\mathrm{n}=1)$

Gall (Fig. 29): on stem, globoid, green, glabrous, one-chambered. Galler: Undetermined. Other insects: Formicidae (Hymenoptera) (predator). Site: BEP (RP), August/2011. New gall morphotype. Previous records on Derris Lour: Santos et al. 2011, on Derris guilleminiana (Tul.) J.F. Macbr. (heterotypic synonym of Lonchocarpus cultratus (Vell.) A. M. G.Azevedo \& H. C. Lima).

Inga ingoides (Rich.) Willd. $(\mathrm{n}=2)$

Gall (Fig. 30): on leafvein (abaxial surface), fusiform, green, glabrous, one-chambered. Galler: Cecidomyiidae (Diptera). Other insects: Eulophidae sp. 2 (Hymenoptera) (parasitoid). Site: BEP (RP), August/2011.

Gall (Fig. 31): on leaf (abaxial surface), lenticular, brown, glabrous, one-chambered. Galler: Hemiptera. Site: BEP (RP), August/2011. Previous records on this plant species: Fernandes et al. 1988, Belo Horizonte (MG).

Inga sp. $(\mathrm{n}=1)$

Gall (Fig. 32): on leaf (abaxial surface), globoid, brown, glabrous. Galler: Undetermined. Other insects: Braconidae sp. 2 (Hymenoptera) (parasitoid). Site: SBF (FC), August/2011. Previous records on undetermined species of Inga Mill.: Maia 2013, São Tomé das Letras (MG), Araújo et al. 2012, Almada and Fernandes 2011, Oriximiná (PA), Maia et al. 2014, Santa Teresa (ES).

Mimosa tweedieana Barneby ex Glazier and Mackinder (new record of host plant species) $(n=1)$ 
Gall (Fig. 33): on stem, globoid, reddish, pubescent, multichambered. Galler: Undetermined. Other insects: Lepidoptera and Coccoidea (Hemiptera). Site: BEP (RP), December/2011.

Zygia latifolia var. communis Barneby and J.W.Grimes $(\mathrm{n}=1)$

Gall (Fig. 34): on leaf (abaxial surface), globoid, pubescent, green, one-chambered. Galler: Undetermined. Other insects: Hymenoptera (parasitoid). Site: BEP (RP), August/2011. New gall morphotype. Previous records on this plant species: Almada and Fernandes 2011, Oriximiná (PA). New gall morphotype.

Lamiaceae Martinov ( $\mathrm{n}=2)$

Hyptis sp. $(\mathrm{n}=1)$

Gall (Fig. 35): on stem, globoid, green, glabrous, one-chambered. Galler: Neolasioptera sp. (Cecidomyiidae, Diptera). Site: SBF (FC), April/2012. Previous records em Hyptis Jacq.: Carneiro et al. 2009, Cadeia do Espinhaço (MG), Fernandes et al. 1997, Vale do Jequitinhonha (MG), Maia and Fernandes 2004, Serra de São José, (MG), Carneiro et al. 2009, Serra do Cipó (MG), Gonçalves-Alvim and Fernandes 2001, Três Marias (MG).

Vitex schomburgkiana Schauer (new record of host plant species) $(\mathrm{n}=1)$

Gall (Fig. 36): on leaf (adaxial surface), conical, green, pubescent, one-chambered. Galler: Undetermined. Site: BEP (RP), December/2011.

Malpighiaceae Juss. $(\mathrm{n}=2)$

Byrsonima sp. $(\mathrm{n}=2)$

Gall (Fig. 37): on stem, globoid, brown, glabrous, multichambered. Galler: Undetermined. Other insects: Hymenoptera (parasitoid). Site: BEP (RP), August/2011.

Gall (Fig. 38): on leaf (abaxial and adaxial surfaces), conical, green, glabrous, one-chambered. Galler: Cecidomyiidae (Diptera). Other insects: Thysanoptera (inquilines). Site: BEP (RP), April/2012.

Meliaceae A. Juss. ( $\mathrm{n}=1)$
Trichilia quadrijuga Kunth ( $\mathrm{n}=1)$

Gall (Fig. 39): on leaf (abaxial and adaxial surfaces), lenticular, green, pubescent, onechambered. Galler: Undetermined. Other insects: Lestodiplosini (Cecidomyiidae, Diptera) (predator). Site: BEP (RF), December/2011. Previous records on this plant species: Santos et al. 2012b, Pernambuco. New gall morphotype.

Moraceae Gaudich. $(\mathrm{n}=2)$

Ficus sp. $1(\mathrm{n}=1)$

Gall (Fig. 40): on leaf, lenticular, brown, glabrous, one-chambered. Galler: Cecidomyiidae (Diptera). Other insects: Eulophidae sp. 3 (Hymenoptera) (parasitoid). Site: BEP (RP), August/2011.

Ficus sp. $2(\mathrm{n}=1)$

Gall (Fig. 41): on leaf, lenticular, yellow, glabrous, one-chambered. Galler: Undetermined. Other insects: Callimicra sp. (Buprestidae, Coleoptera). Site: SBF (FC), April/2012. Previous records on Ficus L.: Maia et al. 2008, on F. enormis (Mart. ex. Miq.) Miq., Bertioga (SP), Julião et al. 2002, on F. gomelleira Kunth, Corumbá (MS), Almada and Fernandes 2011, on F. gomelleira, Oriximiná (PA).

Myrtaceae Juss. ( $\mathrm{n}=13)$

Calyptranthes nigricans DC. (new record of host plant species) $(\mathrm{n}=1)$

Gall (Fig. 42): on leaf, marginal roll, green, glabrous, one-chambered. Galler: Neolasioptera sp. (Cecidomyiidae, Diptera). Site: SBF (FC), August/2011.

Calyptranthes sp. $(\mathrm{n}=1)$

Gall (Fig. 43): on leaf (abaxial and adaxial surfaces), lenticular, brown, glabrous, onechambered. Galler: Cecidomyiidae (Diptera). Sites: SBF (FC) and BEP (RP), April/2012.

Eugenia flavescens DC. (new record of host plant species) $(\mathrm{n}=3)$

Gall (Fig. 44): on stem, globoid, brown, glabrous, one-chambered. Galler: Cecidomyiidae (Diptera). Site: BEP (RP), August/2011. 

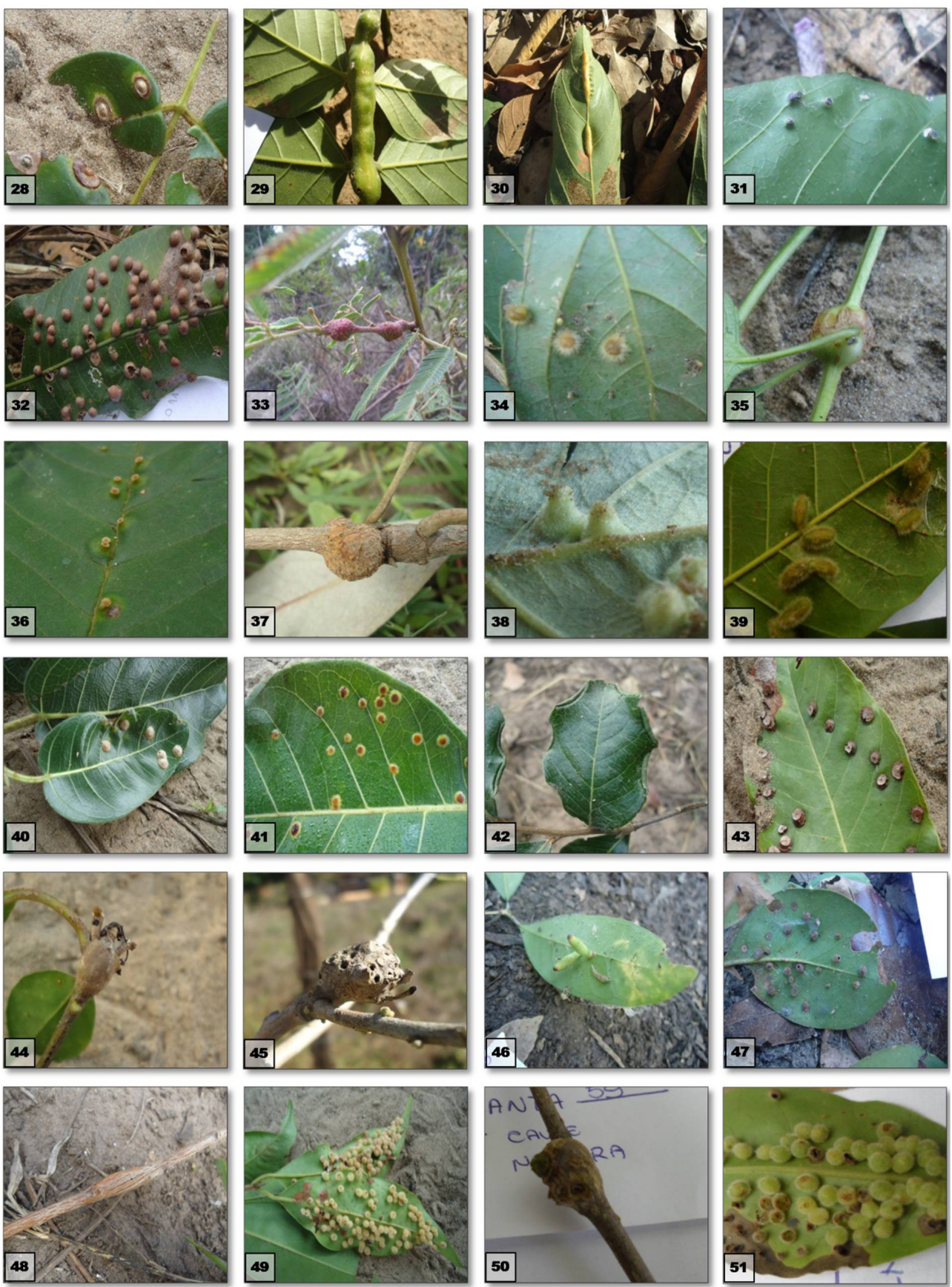

Figures 28-51 - (28) On Copaifera sp.: lenticular leaf; (29) On Derris sp.: globoid stem gall; (30-31) On Inga ingoides: (30) fusiforme leafvein gall; (31) lenticular leaf gall; (32) On Inga sp.: globoid leaf gall; (33) On Mimosa tweedieana: globoid stem gall; (34) On Zygia latifolia var. communis: globoid leaf gall; (35) On Hyptis sp.: globoid stem gall; (36) On Vitex schomburgkiana: conical leaf gall; (37-38) On Byrsonima sp.: (37) globoid stem gall; (38) conical leaf gall; (39) On Trichilia quadrijuga: lenticular leaf gall; (40) On Ficus sp. 1: lenticular leaf gall; (41) On Ficus sp. 2: lenticula leaf gall; (42) On Calyptranthes nigricans: marginal roll leaf gall; (43) On Calyptranthes sp.: lenticular leaf gall; (44-46) On Eugenia flavescens: (44) globoid stem gall; (45) globoid stem gall; (46) cylindrical leaf gall; (47-48) On Eugenia sp. 1: (47) globoid leaf gall; (48) fusiform stem gall; (49) On Eugenia sp. 2: globoid leaf gall; (50) On Marlierea sp.: globoid stem gall; (51) On Myrcia sp.: globoid leaf gall. 
Gall (Fig. 45): on stem, globoid, brown, glabrous, multichambered. Galler: Coleoptera. Site: BEP (RP), August/2011.

Gall (Fig. 46): on leaf (adaxial surface), cylindrical, green, glabrous, one-chambered. Galler: Cecidomyiidae (Diptera). Site: BEP (RP), August/2011.

Eugenia sp. $1(\mathrm{n}=3)$

Gall (Fig. 47): on leaf (adaxial surface), globoid, brown, glabrous, one-chambered. Galler: Undetermined. Site: BEP (RP), August/2011.

Gall (no figures): on stem, fusiform, brown, glabrous, one-chambered. Galler: Undetermined. Site: BEP (RP), August/2011.

Gall (Fig. 48): on stem, fusiform, brown, glabrous, multichambered. Galler: Neolasioptera sp. (Cecidomyiidae, Diptera). Site: BEP (RP), August/2011.

Eugenia sp. $2(\mathrm{n}=1)$

Gall (Fig. 49): on leaf (abaxial surface), globoid, yellow, pubescent, one-chambered. Galler: Cecidomyiidae (Diptera). Site: BEP (RP), April/2012. Previous records on Eugenia spp.: Maia and Fernandes 2004, Serra de São José (MG), Araújo et al. 2011, Serra dos Pireneus (GO). Previous records on; Eugenia spp.: GonçalvesAlvim and Fernandes 2001, Três Marias (MG), Lima et al. 2000, on E. stigmatosa DC., Bertioga (SP), Julião et al. 2002, Corumbá (MS), Maia and Oliveira 2010, Ilha de Cabo Frio (RJ), Araújo et al. 2011, Serra dos Pireneus (GO), Coelho et al. 2009, on E. sonderiana O. Berg, Serra do Cipó (MG), Coelho et al. 2013, on E. punicifolia (Kunth) DC., Serra do Cabral (MG), Maia 2013, on E. biflora L., São Tomé das Letras (MG), Rodrigues et al. 2014, Ilha da Marambaia (RJ).

\section{Marlierea $\mathrm{sp} .(\mathrm{n}=1)$}

Gall (Fig. 50): on stem, globoid, brown, glabrous, one-chambered. Galler: Cecidomyiidae (Diptera). Other insects: Hymenoptera (parasitoid). Site: BEP (RP), December/2011. Previous records on Marlierea Cambess.: Maia 2014, Itamonte (MG).

\section{Myrcia sp. $(\mathrm{n}=1)$}

Gall (Fig. 51): on leaf (abaxial surface), globoid, green, pubescent, one-chambered. Galler: Undetermined. Other insects: Pteromalidae sp. 1 and Platygastridae sp. (Hymenoptera) (parasitoids). Site: BEP (RP), December/2011. Previous records on undetermined species Myrcia DC.: Fernandes et al. 2001, Vale do Rio Doce (MG), Santos et al. 2011, Pernambuco, Maia 2013, São Tomé das Letras, Maia 2014, Itamonte (MG), Maia and CarvalhoFernandes 2016, São Francisco de Itabapoana (RJ), Maia et al. 2014, Santa Teresa (ES).

\section{Psidium sp. $(\mathrm{n}=2)$}

Gall (Fig. 52): on leaf (abaxial surface), conical, yellow, glabrous, one-chambered. Galler: Undetermined. Other insects: Hymenoptera (parasitoid). Site: BEP (RP), April/2012.

Gall (Fig. 53): on leafvein (abaxial surface), fusiform, yellow, glabrous, one-chambered. Galler: Undetermined. Other insects: Hymenoptera (parasitoid). Site: BEP (RP), April/2012. Previous records on Psidium L.: Tavares 1921, on Psidium sp., Bahia, Fernandes et al. 2001, on Psidium guineense Sw., Vale do Rio Doce (MG), Maia et al. 2008, Maia and Oliveira 2010, Carvalho-Fernandes et al. 2016, on Psidium cattleianum Sabine, in Bertioga (SP), Angra dos Reis (RJ), and São João da Barra (RJ), respectively, Julião et al. 2002, on P. guineensis Sw. and Psidium sp., Corumbá (MS), Santos et al. 2011, on P. guineensis Sw. (PE), Rodrigues et al. 2014, on Psidium guajava L. Mangaratiba (RJ).

Phyllanthaceae Martinov ( $\mathrm{n}=1)$

Phyllanthus sp. $(\mathrm{n}=1)$

Gall (no figures): on stem, fusiform, brown, glabrous, multichambered. Galler: Undetermined. Site: BEP (RP), August/2011. Previous records on undetermined species of Phyllanthus L.: Maia et al. 2014, Santa Teresa (ES), Maia and CarvalhoFernandes 2016, RJ. New gall morphotype. 
Polygonaceae A. Juss. ( $\mathrm{n}=8)$

Coccoloba diversifolia Jacq. (new record of host plant species) $(\mathrm{n}=1)$

Gall (Fig. 54): on leaf (abaxial surface), conical, green, glabrous, one-chambered. Galler: Undetermined. Site: BEP (RP), December/2011.

Coccoloba sp. $1(\mathrm{n}=2)$

Gall (Fig. 55): on leaf (abaxial surface), lenticular, brown, glabrous, one-chambered. Galler: Lopesiini (Cecidomyiidae, Diptera). Other insects: Eurytomidae sp. 1 and Eulophidae sp. 4 (Hymenoptera) (parasitoids). Site: BEP (RP), August/2011.

Gall (Fig. 56): on leaf (abaxial surface), globoid, glabrous, brown, one-chambered. Galler: Cecidomyiidae (Diptera). Site: BEP (RP), August/2011.

Coccoloba sp. $2(\mathrm{n}=2)$

Gall (Fig. 57): on leaf (abaxial surface), globoid, green, glabrous, one-chambered. Galler: Cecidomyiidae (Diptera). Other insects: Eurytomidae sp. 2 and Eupelmidae sp. 2 (Hymenoptera) (parasitoids). Site: BEP (RP), December/2011.

Gall (Fig. 58): on leaf (abaxial and adaxial surfaces), lenticular, brown, glabrous, onechambered. Galler: Undetermined. Site: BEP (RP), December/2011.

Coccoloba sp. $3(\mathrm{n}=3)$

Gall (Fig. 59): on leaf (abaxial surface), lenticular, green, glabrous, one-chambered. Galler: Cecidomyiidae (Diptera). Other insects: Hymenoptera (parasitoid). Site: SBF (FC), Apri1/2012.

Gall (Fig. 60): on leaf (abaxial surface), globoid, brown, pubescent, one-chambered. Galler: Cecidomyiidae (Diptera). Site: SBF (FC), April/2012.

Gall (Fig. 61): on bud, globoid, brown, pubescent, solid. Galler: Undetermined. Site: SBF (FC), April/2012. New gall morphotype.
Previous records on undetermined species of Coccoloba P. Browne: Maia et al. 2008, Bertioga (SP), Santos et al. 2012b, (PE).

Rutaceae A. Juss. ( $\mathrm{n}=1)$

Zanthoxylum sp. $(\mathrm{n}=1)$

Gall (Fig. 62): on leaf (abaxial surface), lenticular, yellow, glabrous, one-chambered. Galler: Hemiptera. Site: BEP (RP), August/2011. Previous records on Zanthoxylum L.: Fernandes et al. 1997, Jequitinhonha (MG).

Salicaceae Mirb. $(\mathrm{n}=2)$

Casearia aculeata Jacq. $(\mathrm{n}=1)$

Gall (Fig. 63): on leaf (abaxial and adaxial surfaces), lenticular, green, glabrous, onechambered. Galler: Cecidomyiidae (Diptera). Site: BEP (RP), August/2011. Previous record on this plant species: Fernandes and Negreiros 2006, Aimorés (MG).

Salix humboldtiana Willd. (new record of host plant species) $(\mathrm{n}=1)$

Gall (Fig. 64): on leaf (adaxial surface), globoid, yellow, glabrous, multichambered. Galler: Undetermined. Other insects: Eulophidae sp. 5 and Eulophidae sp. 6 and Sycophila sp. (Eurytomidae) (Hymenoptera) (parasitoids). Site: BEP (RP), August and December/2011 and April/2012.

Sapindaceae Juss. $(\mathrm{n}=09)$

Paullinia ingifolia Rich. ex Juss. (= Paullinia pachycarpa Benth.) (n=3)

Gall (Fig. 65): on leaf (abaxial surface), globoid, green, pubescent, one-chambered. Galler: Schizomyiina (Cecidomyiidae, Diptera). Other insects: Eurytomidae sp. 3, Braconidae sp. 3 (Hymenoptera) (parasitoids) and Tanaostigmoides sp. 1 (Tanaostigmatidae, Hymenoptera) (inquiline). Site: BEP (RP), August/2011 and April/2012. New gall morphotype.

Gall (Fig. 66): on leafvein (abaxial surface), fusiform, green, glabrous, one-chambered. Galler: Undetermined. Site: BEP (RP), April/2012. New gall morphotype. 

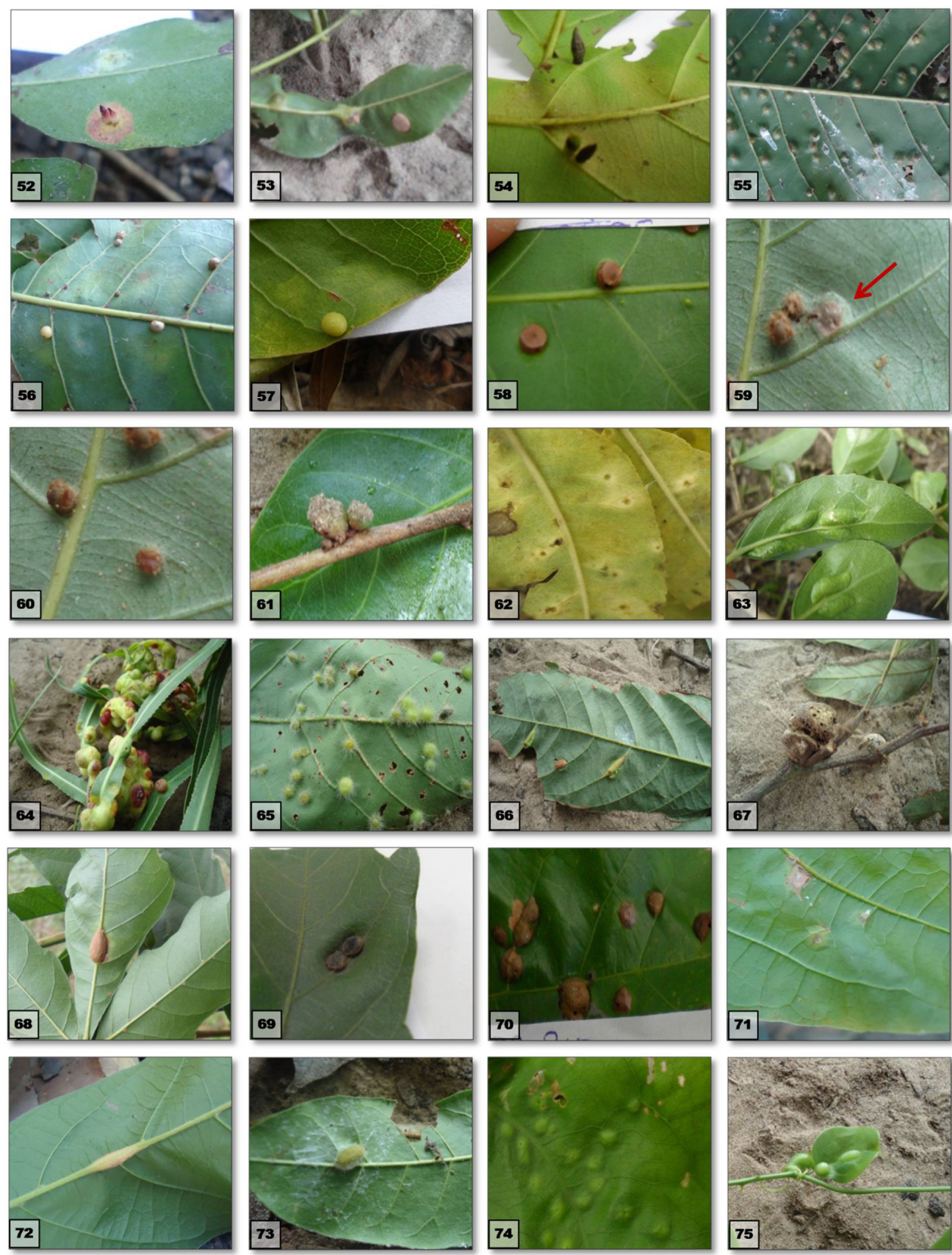

Figures 52-75 - (52-53) On Psidium sp.: (52) conical leaf gall; (53) fusiform leafvein gall; (54) On Coccoloba diversifolia: conical leaf gall; (55-56) On Coccoloba sp. 1: (55) lenticular leaf gall; (56) globoid leaf gall; (57-58) On Coccoloba sp. 2: (57) globoid leaf gall; (58) lenticular leaf gall; (59-61) On Coccoloba sp. 3: (59) lenticular leaf gall; (60) globoid leaf gall; (61) globoid bud gall; (62) On Zanthoxylum sp.: lenticular leaf gall; (63) On Casearia aculeata: lenticular leaf gall; (64) On Salix humboldtiana: globoid leaf gall; (65-67) On Paullinia ingifolia: (65) globoid leaf gall; (66) fusiform leafvein gall; (67) globoid bud gall; (68-72) On Paullinia pinnata: (68) fusiform leafvein gall; (69) globoid leaf gall; (70) lenticular leaf gall; (71) lenticular leaf gall; (72) fusiform leafvein gall; (73) On Vouarana sp.: globoid leaf gall; (74) On Smilax brasiliensis: lenticular leaf gall; (75) On Smilax irrorata: globoid leaf gall. 
Gall (Fig. 67): on bud, globoid, brown, glabrous, multichambered. Galler: Undetermined. Site: BEP (RP), April/2012. New gall morphotype.

Previous records on the same plant species: Araújo et al. 2012, Oriximiná (PA) (as Paullinia pachycarpa Benth.).

Paullinia pinnata L. $(\mathrm{n}=5)$

Gall (Fig. 68): on leafvein (abaxial and adaxial surfaces), fusiform, brown, glabrous, multichambered. Galler: Undetermined. Other insects: Hymenoptera (parasitoid). Site: BEP (RP), December/2011.

Gall (Fig. 69): on leaf (abaxial and adaxial surfaces), globoid, black, glabrous, multichambered. Galler: Cecidomyiidae (Diptera). Site: BEP (RP), December/2011. New gall morphotype.

Gall (Fig. 70): on leaf (adaxial surface), lenticular, brown, glabrous, one-chambered. Galler: Undetermined. Other insects: Eulophidae sp. 7 (Hymenoptera) (parasitoid). Site: BEP (RP), December/2011. New gall morphotype.

Gall (Fig. 71): on leaf (abaxial surface), lenticular, green, glabrous, one-chambered. Galler: Cecidomyiidae (Diptera). Other insects: Hemiptera (inquiline). Site: BEP (RP), August/2011.

Gall (Fig. 72): on leafvein (abaxial surface), fusiform, green, glabrous, one-chambered. Galler: Cecidomyiidae (Diptera). Site: BEP (RP), August/2011. Previous records on the same plant species: Julião et al. 2002, Corumbá (MS)

Vouarana sp. (new record of host plant genus) $(\mathrm{n}=1)$

Gall (Fig. 73): on leaf (abaxial surface), globoid, green, pubescent, one-chambered. Galler: Cecidomyiidae (Diptera). Other insects: Lepidoptera and Trotteria sp. (Cecidomyiidae, Diptera) (inquiline). Site: BEP (RP), August/2011.

Smilacaceae Vent. $(\mathrm{n}=3)$

Smilax brasiliensis Spreng. $(\mathrm{n}=1)$

Gall (Fig. 74): on leaf (abaxial and adaxial surfaces), lenticular, green, glabrous, onechambered. Galler: Cecidomyiidae (Diptera). Site:
BEP (RP), December/2011. Previous records: Carneiro et al. 2009, Cadeia do Espinhaço (MG).

Smilax irrorata Mart. ex Griseb. (new record of host plant species) $(\mathrm{n}=1)$

Gall (Fig. 75): on leaf (abaxial surface), globoid, green, glabrous, one-chambered. Galler: Undetermined. Other insects: Eurytomidae sp. 4 and Eupelmidae sp. 3 (Hymenoptera) (parasitoids). Site: BEP (RP), December/2011.

\section{Smilax sp. $(\mathrm{n}=1)$}

Gall (Fig. 76): on leaf (abaxial surface), lenticular, yellow, glabrous, one-chambered. Galler: Cecidomyiidae (Diptera). Other insects: Eulophidae sp. 8 (Hymenoptera) (parasitoid). Site: BEP (RP), August/2011. Previous records on undetermined species of Smilax L.: Tavares 1909, São Leopoldo (RS), Coelho et al. 2009, Serra do Cipó (MG), Araújo et al. 2011, Serra dos Pireneus (GO), Malves and Frieiro-Costa 2012, Ingaí (MG), Luz et al. 2012, Januária (MG), Maia et al. 2014, Santa Teresa (ES), Araújo et al. 2015 (GO).

Verbenaceae J.St.-Hil $(\mathrm{n}=1)$

Lippia alba (Mill.) N.E.Br. ex Britton and P.Wilson (new record de host plant) $(n=1)$

Gall (Fig. 77): on leaf (adaxial surface), globoid, green, pubescent, one-chambered. Galler: Clinodiplosini (Cecidomyiidae, Diptera). Other insects: Pteromalidae sp. 2 (Hymenoptera) (parasitoid). Site: BEP (RP), August/2011.

\section{DISCUSSION}

Comparing our data with those of Julião et al. (2002) and Urso-Guimarães et al. (2017), we can observe differences in relation to the gall richness and host plant composition. Julião et al. (2002) reported 133 gall morphotypes on 75 plant species and pointed out Bignoniaceae, Fabaceae, Sapindaceae and Hippocrateaceae as the plant families with the greatest number of galled species and gall morphotypes. Urso-Guimarães et al. (2017) recorded 32 gall morphotypes on 23 
plant species and pointed out Sapindaceae as the superhost plant family. In our survey, we found 76 gall morphotypes on 52 plant species and we indicated Fabaceae, Myrtaceae and Sapindaceae as the superhosts. These differences can be attributed to differences in the sample efforts and collection methodology. Julião et al. (2002) adopted the methodology of randomly walking and surveyed 27 sites, totaling 54 hours of collection. UrsoGuimarães et al. (2017) sampled two sites, where a $10 \mathrm{~m}$ wide transect was staked out. Each site was sampled once, totaling two hours of sampling effort. In our survey, we adopted the same methodology of Julião et al. (2002) and investigated three sites, totaling 72 hours. So our sample efforts were done for a longer period of time than that of Julião et al. (2002), but we investigated a smaller extension of area. These data reinforce the impact of the sample efforts in the gall richness. Nevertheless, Fabaceae and Sapindaceae were pointed out previously as an important host plant family. In fact, several Brazilian inventories in different biomes confirm the superhost status of Fabaceae (as for example: Santos et al. 2011 - Caatinga, Luz et al. 2012 transition area of Caatinga-Cerrado, Coelho et al. 2009 - Cerrado, Maia et al. 2014 - Atlantic Forest). The high gall richness of Fabaceae can be explained by the plant richness hypothesis (Southwood 1960, 1961), which predicts that the richest plant taxa comprise the greatest insect gall richness.

Three endemic species were found as host plants and they comprised together six gall morphotypes. Considering that almost all insect gallers are monophagous and each species induces a gall that is anatomically and physiologically different from those induced by other related species (Shorthouse et al. 2005), there is a great probability that these gallers are endemic too.

The mean number of gall morphotypes per plant morphospecies in our study was 1.46. The values recorded by Julião et al. (2002) and UrsoGuimarães et al. (2017) were 1.77 and 1.39,
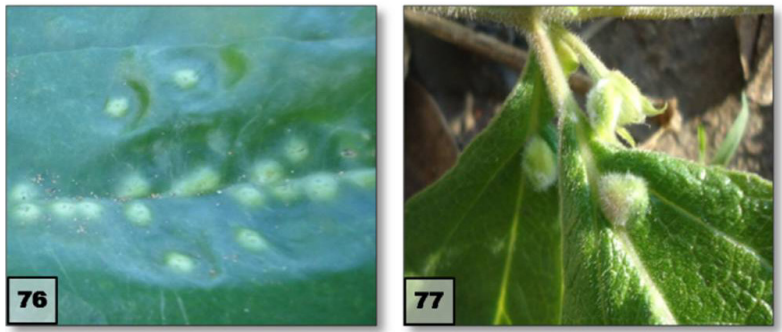

Figures 76-77 - (76) On Smilax sp.: lenticular leaf gall; (77) On Lippia alba: globoid leaf gall.

respectively. Previous studies on insect galls in Brazilian biomes have reported values ranging from 2.1 to 1.1 (Maia et al. 2014). So our result fits into the known range. Most host plant species comprise a single gall morphotype (see Maia 2011 - Amazonian forest, Maia 2001 - Atlantic forest, Santos et al. 2011 - Caatinga, and Carneiro et al. 2009 - Cerrado), but the presence of superhost species increases this mean. In our inventory, Paullinia pinnata L. presented the highest number of gall morphotypes, five, and can be indicated as superhost species. This plant together with two others (Paullinia ingifolia Rich. ex Juss. and Andira inermis) helped to increase this mean (without them, the mean decreases to 1.31).

About $76.3 \%$ of the gall morphotypes were observed on leaves. The highest frequency of leaf galls is an already known world pattern. Most galls were globoid, green or brown, glabrous and onechambered. These features have been indicated in several Brazilian biomes as the most common, suggesting a widespread pattern. Nevertheless, Julião et al. (2002) pointed out the predominance of fusiform galls. Diptera (Cecidomyiidae) were the most frequent gallers. The same result was found by Julião et al. (2002), but differing from these authors, we did not find Hymenopteran and Lepidopteran galls. As Urso-Guimarães et al. 2017 identified the inducer of a single gall morphotype, we cannot compare the results. Cecidomyiidae are in the entire world the most frequent galling insects (Gagné and Jaschhof 2014). 
Among the cecidomyiids, we recorded the occurrence of Neolasioptera Felt 1908 and Trotteria. The former is known from the Pantanal by a single newly described species, Neolasioptera pantaneira Maia, 2017 (Maia et al. 2017), the latter is reported for the first time in this biome.

In our study, the parasitoids were the major component of the associated fauna, as in other Brazilian inventories (Maia 2001, Maia and Fernandes 2004, Maia et al. 2008). They were represented by Eulophidae, Braconidae, Eupelmidae, Eurytomidae, Platygastridae and Pteromalidae, families already recorded as parasitoids of galling insects in Brazil (Maia and Azevedo 2009). Eulophidae were the most frequent one, as previously pointed out by Maia and Azevedo 2009 at restinga areas (Atlantic forest). Multiparatism has already been recorded in galls of the Atlantic forest (Maia 1999, Maia and Monteiro 1999, Maia and Tavares 2000, Maia and Azevedo 2009), and Cerrado (Fernandes et al. 1997, Maia and Fernandes 2004), but not in the Pantanal.

The percentage of parasitized leaf galls was higher than that of stem galls. This result suggests that the galling larvae are more protected against parasitoids in the stem than in leaf galls. The tissue hardness can possibly explain it (Levin 1973, Southwood 1986), as stem galls are harder than leaf galls. According to Craig et al. 1990, the hardening of gall walls prevents penetration of the ovipositor of parasitoid wasps that would reach the larva and inhibits feeding on the gall tissue by herbivores. Another gall feature that can affect the rate of parasitism is the presence of trichomes. According to Richardson (1943), plant trichomes can act as structural defenses as they impair feeding and mobility. In the present work, we observed similar percentages of parasitized glabrous galls (30.1\%) and parasitized pubescent galls (30.8\%), so our results cannot confirm the trichomes' protective function.
Data on gall inquilines and predators are scarce in Brazil, as few studies with this focus have been published. Nevertheless, the taxonomic taxa observed in our study have already been recorded (Maia 1995, 2001, Maia et al. 2008), except Tanaostigmoides (Hymenoptera) which is recorded for the first time here. It is interesting to emphasize that records of pseudoscorpions in galls are known only in Brazil and Africa (Maia 2001, Maia and Souza 2013, Rodrigues et al. 2014, Maia 2013, Carvalho-Fernandes et al. 2012, Judson and Heurtault 1996). Although their association with galls on Fabaceae is previously known, this is the first record of pseudoscorpions in galls on Andira inermis (W.Wright) Kunth ex DC (Fabaceae). Besides, Olpiolum medium Beier, 1931 is reported for the first time in insect galls.

Callimicra sp. (Coleoptera, Buprestidae) was found in a lenticular gall on Ficus sp. (Moraceae). In the Neotropical Region, records of Buprestidae in galls are scarce, as well as in the entire world (Maia 2012, Bellamy and Scholtz 1986). According to Hespenheide 2013, larvae of most species of Callimicra Deyrolle, 1864 are phytophagous and live on their host plants. Hanson (2009) recorded species of Callimicra in association with Ficus hirsuta Schott (Moraceae) and Ficus sp. We cannot affirm if this beetle is galler, but as it was the single dweller obtained from a gall without evidences of invasion, we believe that this can be the first record of Buprestidae galler in Brazil.

As in Julião et al. (2002) and Urso-Guimarães et al. (2017) there is no record of the associated fauna, our data represent the first contribution to the its knowledge.

The BEP comprised higher gall richness than the SBF, but the sample effort was greater in the former than in the latter. Nevertheless, some patterns were observed in both localities: leaves were the most galled plant organ; there was a predominance of globoid galls, brown and green colors, glabrous and one-chambered galls; and cecidomyiids were 
the most frequent galling insects. Parasitoids were found in both areas, but they were represented by different species.

In spite of being the third study about insect galls of the Pantanal, several new records were pointed out, showing that the gall diversity of this biome can be greater than expected. Although the harsh environment hypothesis which predicts that insect galls are especially abundant in plant species found in hot and dry habitats (Fernandes and Price 1988, 1992) has not been tested, the present inventory does not add data in favor of it. Studies with this approach are necessary to confirm or reject this hypothesis.

The taxonomy of the inducers is other important challenge as most part is still superficially identified or is unknown. It requires a long-term research with frequent collections to follow the entire lifecycle of the gallers and obtain all stages necessary to their identification.

\section{REFERENCES}

ALMADA ED AND FERNANDES GWA. 2011. Insetos indutores de galhas em florestas de terra firme e em reflorestamentos com espécies nativas na Amazônia Oriental, Pará, Brasil. Bol Mus Para Emílio Goeldi. Ciênc Nat 6(2): 163-196.

ARAÚJO WS, PORFÍRIO-JUNIOR ED, JORGE VA AND ESPÍRITO-SANTO FILHO K. 2012. Plantas hospedeiras e galhas entomógenas em sub-bosques de florestas tropicais do Pará, Brasil. Insula 41: 59-72.

ARAÚJO WS, PORFÍRIO JUNIOR ED, RIBEIRO BA, SILVA TM, SILVA EC, GUILHERME FAG, SCARELISANTOS C AND SANTOS BB. 2015. Checklist of host plants of insect galls in the State of Goiás in the Midwest region of Brazil. Biodivers Data J 3: e6835.

ARAÚJO WS, SANTOS BB, FERREIRA HD AND LOUSA TC. 2007. Ocorrência de galhas entomógenas na vegetação do Campus da UFG, em Goiânia, Goiás. Rev Bras Biociênc 5(1): 57-59.

ARAÚJO WS, SANTOS BB AND GOMES-KLEIN VL. 2011. Insect galls from Serra dos Pireneus, GO, Brazil. Biota Neotrop 11(2): 357-365.

BELLAMY CL AND SCHOLTZ CH. 1986. Two new species of gall-forming Sphenoptera solier (Coleoptera: Buprestidae) from South Africa. The Coleopt Bull 40(2): 117-126.
CARNEIRO MAA, BORGES RX, ARAÚJO APA AND FERNANDES GW. 2009. Insetos indutores de galhas da porção sul da Cadeia do Espinhaço, Minas Gerais, Brasil. Rev Bras Entomol 53(4): 570-592.

CARVALHO-FERNANDES SP, ALMEIDA-CORTEZ J AND FERREIRA ALN. 2012. Riqueza de galhas entomógenas em áreas antropizadas e preservadas de caatinga. Rev Árvore 36(2): 269-277.

CARVALHO-FERNANDES SP, ASCENDINO S, MAIA VC AND COURI MS. 2016. Diversity of insect galls associated with coastal shrub vegetation in Rio de Janeiro, Brazil. An Acad Bras Cienc 88: 1407-1418.

COELHO MS, ALMADA ED, FERNANDES GW, CARNEIRO MAA, SANTOS RM, QUINTINO AV AND SANCHEZ-AZOFEIFAA. 2009. Gall inducing arthropods from a seasonally dry tropical forest in Serra do Cipó, Brazil. Rev Bras Entomol 53(3): 404-414.

COELHO MS, CARNEIRO MAA, BRANCO CA AND FERNANDES GW. 2013. Gall-inducing insects from Serra do Cabral, Minas Gerais, Brazil. Biota Neotrop 13(3): 102-108.

CRAIG TP, ITAMI JK AND PRICE PW. 1990. The window of vulnerability of a shoot-galling sawfly to attack by a parasitoid. Ecology 71: 1471-1482.

DREGER-JAUFFRET F AND SHORTHOUSE JD. 1992. Diversity of gall-inducing insects and their galls. In: Shorthouse JD and Rohfritsch O (Eds), Biology of InsectInduced Galls, New York, Oxford University Press, 285 p.

FERNANDES GW, ARAUJO RC, ARAUJO SC, LOMBARDI JA, PAULA AS, LOYOLA JUNIOR R AND CORNELISSEN TG. 1997. Insect galls from savanna and rocky fields of the Jequitinhonha Valley, Mina Gerais, Brazil. Naturalia 22: 221-244.

FERNANDES GW, JULIÃO GR, ARAÚJO RC, ARAÚJO SC, LOMBARDI JA, NEGREIROS D AND CARNEIRO MA. 2001. Distribution and morphology of insect galls the Rio Doce Valley, Brazil. Naturalia 26: 211-244.

FERNANDES GW AND NEGREIROS D. 2006. A comunidade de insetos galhadores da RPPN Fazenda Bulcão, Aimorés, Minas Gerais, Brasil. Lundiana 7(2): 111-120.

FERNANDES GW AND PRICE PW. 1988. Biographical gradients in galling species richness. Test of hypotheses. Oecologia 76: 161-167.

FERNANDES GW AND PRICE PW. 1992. The adaptative significance of insect gall distribution: survivorship of species in xeric and mesic habitats. Oecologia 90: 14-20.

FERNANDES GW, TAMEIRÃO NETO ET AND MARTINS RP. 1988. Ocorrência e caracterização de galhas entomógenas do Campus Pampulha da Universidade Federal de Minas Gerais. Rev Bras Zool 5(1): 11-29.

GAGNÉ RJ. 1994. The gall midges of the Neotropical region. Ithaca, Cornell University Press, 352 p. 
GAGNÉ RJ AND JASCHHOF M. 2014. A Catalog of the Cecidomyiidae (Diptera) of the World. 3rd Edition. Digital version 2. Available from: $<\mathrm{http}$ ://www.ars.usda. gov/SP2UserFiles/Place/80420580/Gagne_2014_World Cecidomyiidae_Catalog_3rd_Edition.pdf $>$ Acess on 13 Feb. 2015.

GONÇALVES-ALVIM S AND FERNANDES GW. 2001. Comunidades de insetos galhadores (Insecta) em diferentes fisionomias do cerrado em Minas Gerais, Brasil. Rev Bras Zool 18(Supl. 1): 289-305.

HANSON C. 2009. Catalogue of the Eulophidae in the Neotropical region. Memoirs of the American Entomological Institute. Florida 82: 916.

HESPENHEIDE HA. 2013. Two new remarkable Callimicra Deyrolle, 1864 (Coleoptera: Buprestidae: Agrilinae) from Central America. Zootaxa 3635(3): 297-300.

IBGE. 2004. Mapas de biomas e vegetação. Disponível em: <http://www.ibge.gov.br/home/presidencia/ noticias/21052004biomashtml.shtm $>$.Acesso em: $15 \mathrm{de}$ fevereiro de 2017.

JUDSON MLI AND HEURTAULT J. 1996. Nanolpium species (Garypoidea, Olpiidae) on grasses in southern Africa - a new niche for pseudoscorpions. Rev Suisse Zool vol. hors 1: 321-326.

JULIÃO GR, AMARAL MEC AND FERNANDES GW. 2002. Galhas de insetos e suas plantas hospedeiras no pantanal Sul-Mato-Grossense. Naturalia 24: 47-74.

LEVIN DA. 1973. The role of trichomes in plant defense. Q Rev Biol 48: 3-15.

LIMA ES, MAGENTA MAG, KRAUS JE, VECHI C AND MARTINS SE. 2000. Levantamento preliminar de galhas entomógenas ocorrentes em plantas das restingas de Bertioga (SP). Anais do V Simpósio de Ecossistemas Brasileiros: Conservação. ACIESP 109(III): 39-46.

LUZ GR, FERNANDES GW AND SILVA JO. 2012. Galhas de insetos em habitats xérico e mésico em região de transição Cerrado-Caatinga no norte de Minas Gerais, Brasil. Neotrop Biol Conserv 7(3): 171-187.

MAIA VC. 1995. Chaves para classificação de galhas de Cecidomyiidae (Diptera) em Myrtaceae na restinga da Barra de Maricá, Rio de Janeiro. Rev Bras Zool 12(41): 1009-1013.

MAIA VC. 1999. Artrópodes associados às galhas de Cecidomyiidae (Diptera) em Eugenia rotundifolia (Myrtaceae) e Clusia lanceolata (Clusiaceae) no Brasil. Iheringia, Zool 87: 75-79.

MAIA VC. 2001. The gall midges (Diptera, Cecidomyiidae) from three restingas of Rio de Janeiro State, Brazil. Rev Bras Zool 18(2): 305-656.

MAIA VC. 2011. Characterization of insect galls, gall makers, and associated fauna of Platô Bacaba (Porto de Trombetas, Pará, Brazil). Biota Neotrop 11(4): 37-53.
MAIA VC. 2012. Coleopterous galls from the Neotropical region. Pap Avulsos Zool 52(15): 175-184.

MAIA VC. 2013. Insect galls of São Tomé das Letras (MG, Brazil). Biota Neotrop 13(4): 164-189.

MAIA VC. 2014. Insect galls of Itamonte (Minas Gerais, Brazil): characterization and occurrence. Biota Neotrop 14(1): 1-17.

MAIA VC AND AZEVEDO MAP. 2009. Micro-himenópteros associados com galhas de Cecidomyiidae (Diptera) em Restingas do estado do Rio de Janeiro. Biota Neotrop 9: $1-14$.

MAIA VC, CARDOSO JLT AND BRAGA JMA. 2014. Insect galls from Atlantic Forest areas of Santa Teresa, Espírito Santo, Brazil: characterization and occurrence. Bol Mus Biol Mello Leitão, Nova Ser 33: 47-129.

MAIA VC AND CARVALHO-FERNANDES SP. 2016. Insect galls of a protected remnant of the Atlantic Forest tableland from Rio de Janeiro State (Brazil). Rev Brasil Entomol 60: 40-56.

MAIA VC, CATIAN G AND LEME FM. 2017. Neolasioptera pantaneira, a new species of Cecidomyiidae (Diptera) associated with Aeschynomene denticulata (Fabaceae) from Brazil. Braz J Biol 77: 170-175.

MAIA VC AND FERNANDES GW. 2004. Insect galls from Serra de São José (Tiradentes, MG, Brazil). Braz J Biol 64(3A): 423-445.

MAIA VC, MAGENTA MAG AND MARTINS SE. 2008. Ocorrência e caracterização de galhas de insetos em áreas de restinga de Bertioga (São Paulo, Brasil). Biota Neotrop 8: 167-197.

MAIA VC AND MONTEIRO RF. 1999. Espécies cecidógenas (Diptera, Cecidomyiidae) e parasitóides (Hymenoptera) associadas a Guapira opposita (Vell.) Reitz. (Nyctaginaceae) na Restinga da Barra de Maricá, Rio de Janeiro. Rev Brasil Zool 16(2): 483-487.

MAIA VC AND OLIVEIRA JC. 2010. Galhas de insetos da Reserva Biológica Estadual da Praia do Sul (Ilha Grande, Angra dos Reis, RJ). Biota Neotrop 10(4): 227-238.

MAIA VC AND SOUZA MC. 2013. Insect galls of the xeric vegetation of Ilha do Cabo Frio (Arraial do Cabo, RJ, Brazil). Biota Neotrop 13(3): 278-288.

MAIA VC AND TAVARES MT. 2000. Cordiamyia globosa Maia (Diptera, Cecidomyiidae), flutuação populacional e parasitóides (Hymenoptera) associados. Rev Brasil Zool 17(3) : 589-593.

MALVES K AND FRIEIRO-COSTA FA. 2012. List of plants with galls induced by insects from the UNILAVRAS/ Boqueirão Biological Reserve, Ingaí, State of Minas Gerais, Brazil. Checklist 8(3): 426-439.

MANI MS. 1964. The ecology of plant galls. Dr. Junk, The Hague, xii +434 . 
MMA - MINISTERIO DO MEIO AMBIENTE. 2015. Disponível em $<$ http://mma.gov.br/biomas $>$. Acesso em 27 julho de 2015.

MONTEIRO RF, ODA RAM, NARAHARA KL AND CONSTANTINO PAL. 2004. Galhas: Diversidade, Especificidade e Distribuição, p. 127-141. In: Pesquisa de Longa Duração na Restinga de Jurubatiba: Ecologia, História Natural e Conservação. Rocha CFD, Esteves FA and Scarano FR (Orgs), RiMa Editora, São Carlos, 374 p.

OLIVEIRA DAS. 2007. Biodiversidade do Cerrado e Pantanal: áreas e ações prioritárias para conservação / Ministério do Meio Ambiente. Brasília. MMA (17): 540.

OLIVEIRA JC AND MAIA VC. 2005. Ocorrência e caracterização de galhas de insetos na restinga de Grumari (Rio de Janeiro, RJ, Brasil). Arq Mus Nac 63(4): 669-676.

RAMBO PR. 1956. A fisionomia do Rio Grande do Sul. Liv. Selbach, Porto Alegre, $456 \mathrm{p}$.

RICHARDSON H. 1943. The action of bean leaves against the bedbug. J Econ Entomol 36: 543-545.

RODRIGUES AR, MAIA VC AND COURI MS. 2014. Insect galls of restinga areas of Ilha da Marambaia, Rio de Janeiro, Brazil. Rev Brasil Entomol 58(2): 173-197.

SANTOS JC, ALMEIDA-CORTEZ JS AND FERNANDES GW. 2012b. Gall-inducing insects from Atlantic forest of Pernambuco, Northeastern Brazil. Biota Neotrop 12(3): 197-213.

SANTOS JC, RIBEIRO BA, SILVA TM AND ARAUJO WS. 2012a. Galhas de insetos em uma área de cerrado sentido restrito na região semi-urbana de Caldas Novas (Goiás, Brasil). Rev Bras Biociênc 10(4): 439-445.

SANTOS JCA, ALMEIDA-CORTEZ JSB AND FERNANDES GW. 2011. Diversity of gall-inducing insects in the high altitude wetland forests in Pernambuco, Northeastern Brazil. Braz J Biol 71(1): 47-56.

SHORTHOUSE JD, WOOLBD AND ANANTANARAYANAN R. 2005. Gall-inducing insects - Nature's most sophisticated herbivores. Basic Appl Ecol 6: 407-411.

SILVA JSV, ABDON MM, BOOCK A AND SILVA MP. 1998. Fitofisionomias dominantes em parte das subregiões do Nabileque e Miranda, sul do Pantanal. Pesq agropec bras, 33: 1713-1719.
SOUTHWOOD TE. 1960. The abundance of the Hawaiian trees and the number of their associated insect species. Proc Hawaiian Entomol Soc 17: 299-303.

SOUTHWOOD TRE. 1961. The number of insect associated with various trees. J Anim Ecol 30: 1-8.

SOUTHWOOD TRE. 1986. Plant surfaces and insects - an overview, p. 1-22. In: Juniper B and Southwood TRE (Eds), Insects and the plant surface. London, Edward Arnold.

URSO-GUIMARÃES MV, CASTELLO ACD, KATAOKA EY AND KOCH I. 2017. Characterization of entomogen galls from Mato Grosso do Sul, Brazil. Rev Brasil Entomol 61(1): 25-42.

URSO-GUIMARÃES MV AND SCARELI-SANTOS C. 2006. Galls and gall makers in plants from the Pé-deGigante Cerrado Reserve, Santa Rita do Passa Quatro, SP, Braz J Biol 66(1b): 357-369.

URSO-GUIMARÃES MV, SCARELI-SANTOS AND BONIFÁCIO-SILVA AC. 2003. Occurrence and characterization of entomogen galls from natural vegetation areas in Delfinópolis, MG, Brazil. Braz J Biol 63(4): 705-715.

TAVARES JS. 1909. Contributio prima ad cognitionem cecidologiae braziliae. Broter Ser Zool 8: 5-28, pls. I-VIII.

TAVARES JS. 1921. Cecidologia brazileira: cecídias que se criam em plantas das famílias das Leguminosae, Sapotaceae, Lauraceae, Myrtaceae, Punicaceae, Aurantiaceae, Malpighiaceae, Sapindaceae, Umbelliferae, Loranthaceae, Apocynaceae, Urticaceae, Salicaceae e Gramineae. Broter Ser Zool 19 (2): 76-96.

TAVARES JS. 1925. Nova contribuição para o conhecimento da cecidologia brazileira. Broter Ser Zool 22: 5-22, pls. $\mathrm{I}-\mathrm{V}$.

\section{SUPPLEMENTARY MATERIAL}

TABLE SII - List of associated fauna in São Bento Farm (SBF) FC - Forest Capões and Base de Estudos do Pantanal (BEP) RF - Riparian Forest. BEP - Base de Estudos do Pantanal, RF - Riparian Forest, SBF - SãBento Farm, FC - Forest Capões. 